\title{
Development of Magnetic Resonance Technology For Noninvasive Boron Quantification
}

\author{
Kenneth M. Bradshaw
}

Published November 1990

EG\&G Idaho, Inc.

Idaho Falls, Idaho $\mathbf{8 3 4 1 5}$

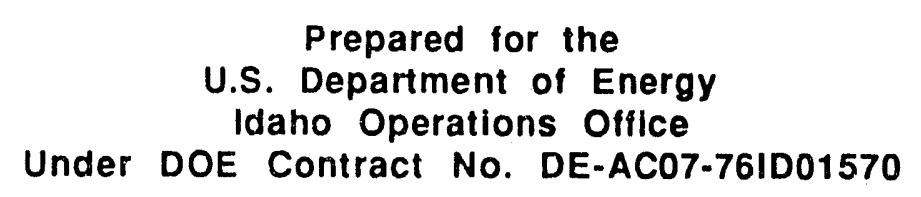




\section{Table of Contents}

Abstract $\ldots \ldots \ldots \ldots \ldots \ldots \ldots \ldots \ldots \ldots \ldots \ldots \ldots \ldots$ i

Acronyms $\ldots \ldots \ldots \ldots \ldots \ldots \ldots \ldots \ldots \ldots \ldots \ldots$

Nomenclature $\ldots \ldots \ldots \ldots \ldots \ldots \ldots \ldots \ldots \ldots \ldots \ldots \ldots$ vi

Background $\ldots \ldots \ldots \ldots \ldots \ldots \ldots \ldots \ldots \ldots \ldots \ldots \ldots \ldots$

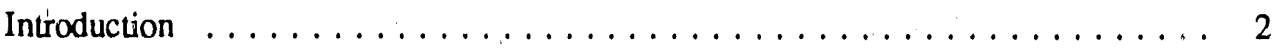

Hardware Requirements $\ldots \ldots \ldots \ldots \ldots \ldots \ldots \ldots \ldots \ldots \ldots \ldots$

RF Power Amplifier . . . . . . . . . . . . . . . . . . . 3

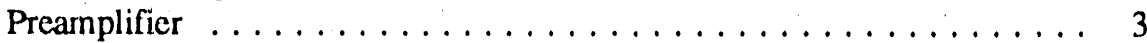

Preamplifier protection $\ldots \ldots \ldots \ldots \ldots \ldots \ldots \ldots \ldots \ldots$

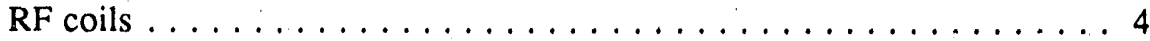

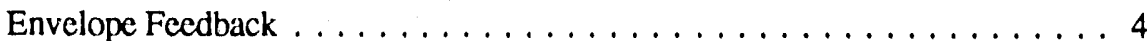

Frequency Source . . . . . . . . . . . . . . . . . . . . . 4

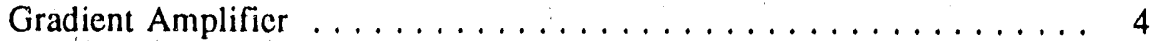

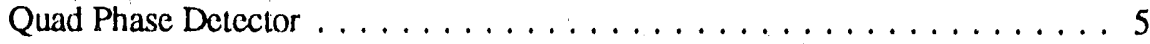

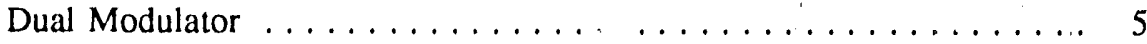

Description of Hardware Mødifications $\ldots \ldots \ldots \ldots \ldots \ldots \ldots \ldots \ldots \ldots$

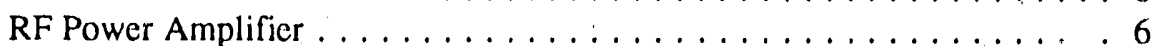

Preamplifier $\ldots \ldots \ldots \ldots \ldots \ldots \ldots \ldots \ldots \ldots \ldots \ldots \ldots$

Preamplifier Protection $\ldots \ldots \ldots \ldots \ldots \ldots \ldots \ldots \ldots$

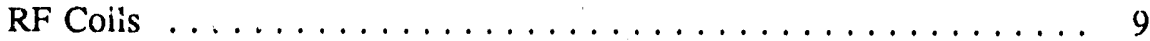

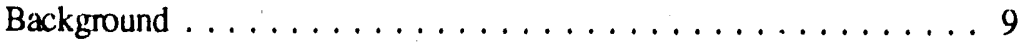

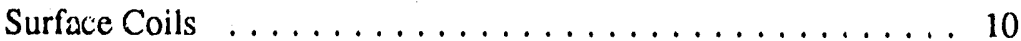

Linear Volurne Coils . . . . . . . . . . . . . . . 11

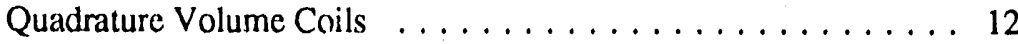

Other Hardware Modifications . . . . . . . . . . . . . . . . 14

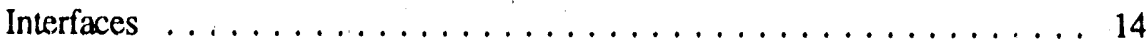

Macintosh Connection . . . . . . . . . . . . . . . 14

Software Development $\ldots \ldots \ldots \ldots \ldots \ldots \ldots \ldots \ldots \ldots \ldots \ldots$

Signa Programs $\ldots \ldots \ldots \ldots \ldots \ldots \ldots \ldots \ldots \ldots \ldots \ldots \ldots \ldots$

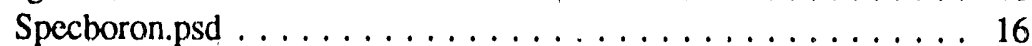

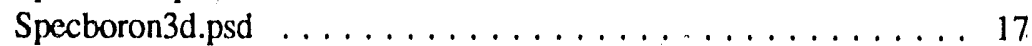

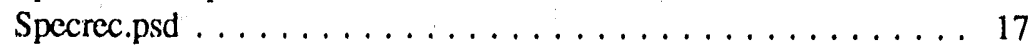

Readsigspec $\ldots \ldots \ldots \ldots \ldots \ldots \ldots \ldots \ldots \ldots \ldots \ldots$

Kermit . . . . . . . . . . . . . . . . . . . . 17

Macintosh Programs . . . . . . . . . . . . . . . . . . 17

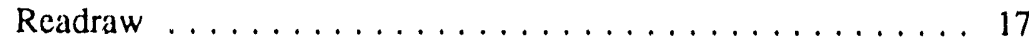

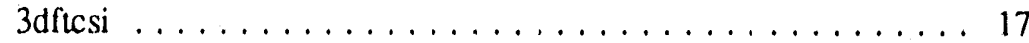

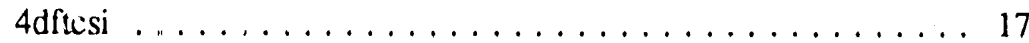

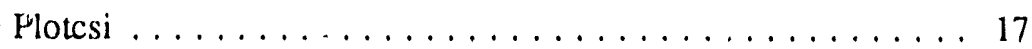

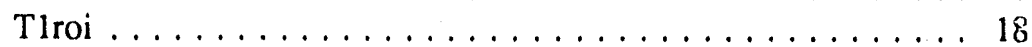

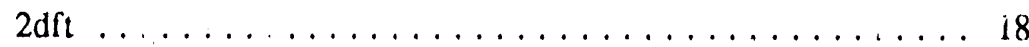


Macft ....................... 18

Operation of the Signa ${ }^{T M}$ MRI System . . . . . . . . . . . . . . . 19

Setup for Boron Operation . . . . . . . . . . . . . . . . . . . . 19

Operation ......................... 19

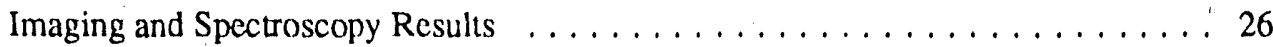

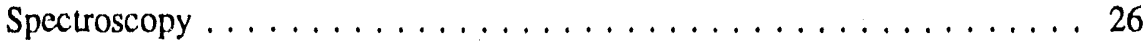

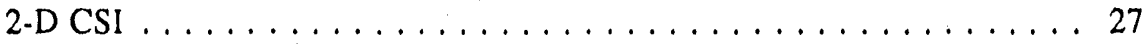

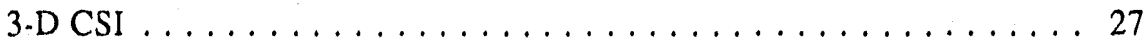

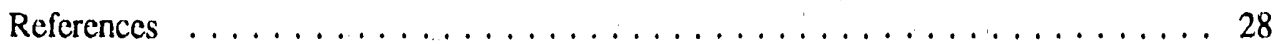

Appendices

Appendix A - Broadband RF Power Amplifier Specification ....... A1

Appendix B - RF Power Amplifier Manual . . . . . . . . . . B1

Appendix $C$ - Macintosh Software Instructions $\ldots \ldots \ldots \ldots \ldots \ldots, C 1$

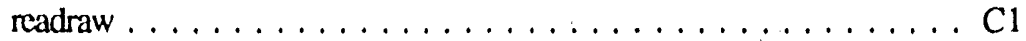

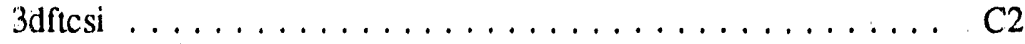

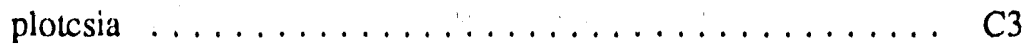

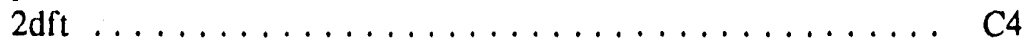

Appendix D - MR Glossary ................. D1 


\section{Acronyms}

$\begin{array}{ll}\text { A/D } & \text { analog to digital } \\ \text { CSI } & \text { chemical shift imaging } \\ \text { DC } & \text { direct current } \\ \text { FID } & \text { free induction decay } \\ \text { FOV } & \text { field of view } \\ \text { MCV } & \text { modify control variables } \\ \text { MR } & \text { magnetic resonance } \\ \text { MRI" } & \text { magnetic resonance imaging } \\ \text { MRS } & \text { magnetic resonance spectroscopy } \\ \text { Nav } & \text { number of averages } \\ \text { NMR } & \text { nuclear magnetic resonance } \\ \text { PPL } & \text { pulse programming language } \\ \text { PSD } & \text { phase sensitive detector or pulse sequence definition } \\ \text { QPD } & \text { quad phase detector } \\ \text { RA } & \text { receiver attentuation } \\ \text { RF } & \text { radio-frequency } \\ \text { SCM } & \text { service control module } \\ \text { SNR,S/N } & \text { signal-to-noise ratio } \\ \text { TA } & \text { transmitter attenuation }\end{array}$




\section{Nomenclature}

$\begin{array}{ll}\text { B } & \text { magnetic field in Tesla }(\mathrm{T}) \text { or gauss }(\mathrm{g}) \\ \mathrm{C} & \text { capacitor, capacitance in Farads }(\mathrm{F}) \\ \mathrm{f} & \text { frequency in Hertz }(\mathrm{Hz}) \text { or cycles per second } \\ \mathrm{G} & \text { gradient magnetic field in gauss }(\mathrm{g}) \\ \mathrm{Hz} & \text { Hertz, cycles per second } \\ \mathrm{L} & \text { inductor, inductance in Henries }(\mathrm{H}) \\ \mathrm{M} & \text { mutual inductance in Henries }(\mathrm{H}) \\ \mathbf{M} & \text { magnetization vector in gauss }(\mathrm{g}) \text { or Tesla }(\mathrm{T}) \\ \mathrm{MHz} & \text { megahertz, million cycles per second } \\ \mathrm{Q} & \text { quality factor } \\ \mathrm{R}, \mathrm{r} & \text { resistor, resistance in ohms }(\Omega) \\ \mathrm{X} & \text { reactance in ohms } \\ \gamma & \text { magnetogyric ratio constant } \\ \omega & \text { frequency in radians, } 2 \pi \mathrm{f}\end{array}$




\section{DEVELOPMENT OF MAGNETIC RESONANCE TECHNOLOGY FOR NONINVASIVE BORON QUANTIFICATION}

\section{Background}

Acquisition of boron pharmacokinetic data in a large animal model study by presently accepted methods requires the sacrificing of the animals, necropsy, and chemical analysis of tissuc for boron content. These methods usually result in only one data point per animal for the time-dependent response function. Determination of tissue kinetic response for a given protocol requires large numbers of samples for statistical analysis to accommodate the effects seen with animal-to-animal biovariability. If there is variation in the established protocol, the same pharmacokinetic experiments must be repeated in order to observe the differences in response. Ideally, the response from variations in protocols is best observed when performed on the same animal. This is usually not possible when using invasive mcasures.

A noninvasive technology, able to detect absolute, or even relative, concentration as a function of time, would offer the opportunity for a dramatic increase in data acquisition rate, as well as providing a major research program cost reduction. With noninvasive techniques, the same animal may be used to observe the results of any experimental procedure change.

If this noninvasive technology is deployed, it will be used to quantitate boron in tumors while doing patient suitability screening and patient treatment planning. For patient treatment planning, the boron distribution in tissue will be used to calculate the tolerable neutron exposure and probability of tumor control.

One noninvasive technique for measuring elemental concentrations presently being investigated is nuclear magnetic resonance (NMR). Magnctic Resonance (MR) is the same as NMR except when NMR was developed into an patient imaging modality the name was changed to alleviate a fear factor with "things nuclear".
Many atomic nuclei have an angular momentum arising from their inherent property of rotation, or spin. Since nuclei are electrically charged, the spin corresponds to a current flowing about the spin axis, which in turn generates a small magnetic field. Each nucleus of nonzero spin therefore has a magnetic moment, or dipole associated with it.

In general, the magnetic dipoles of the nuclei having spin will be pointing in random directions, but when they are placed into an external magnetic field, they will orient themselves with the lines of force either parallel to or antiparallel to the field (in the opposite direction). The two orientations have slightly different energies, described as a Zeeman splitting of the energy levels. With protons the difference between the number of protons with spin "up" (parallel) and spin "down" (antiparallel) is very small: only about one part in $10^{8}$, with a slight excess in the lower energy state (spin up). 1

Since the energy difference is small even for a single nuclei, the magnetic behavior of an entire population of nuclei must be examined on a macroscopic level. The resultant magnetic vector, M, for this entire population is the net effect of all the magnetic moments for a given nuclear species in the sample being examined. Without an external magnetic field this bulk magnetization is zero. When a magnetic field is imposed on the sample, however, the nuclear dipoles become oriented to yield a finite equilibrium bulk magnetization that will point in a direction parallel to the applied magnetic field (conventionally defined as the $\mathrm{Z}$ axis).

Spinning nuclei can be regarded as tiny tops or gyroscopes. Tipping a spinning gyroscope causes a precession around the original axis of motion. In a similar manner, the bulk magnetization, $\mathbf{M}$, corresponding to a population of nuclei, can be tipped away from the $Z$ axis and then will precess around the $Z$ axis. Such tipping can be achieved by applying a much smaller magnetic field that is rotating in the $X$ $Y$ plane at the same frequency of precession. In practice the rotating magnetic field is applied by 
surrounding the sample with a coil connected to a source of radio-frequency (RF) power. This RF power must match the natural precessional frequency of the nuclei, hence the name nuclear magnetic resonance.

A simple mathematical relation links the resonance frequency, often called the Larmor frequency, to the value of the externally applied static magnetic field. Equation (1) relates magnetic ficld to frequency through a magnetogyric ratio $(\gamma)$ which is unique for each nuclear species of nonzero spin.

$f=\gamma B_{0}$

where

$f=$ frequency of precession

$\gamma=$ magnetogyric ratio constant

$\mathrm{B}_{0}=$ static magnetic field in tesla $(\mathrm{T})$.

For nuclei of hydrogen (protons) in a magnetic ficld of one tesla (T) the $\gamma$ is $42.58 \mathrm{MHz} / \mathrm{T}$. For $11 \mathrm{~B}$ and 10 B nuclei, the $\gamma^{\prime} \mathrm{s}$ are 13.66 and $4.576 \mathrm{MHz} / \mathrm{T}$ giving resonant frequencies of 20.49 and $6.86 \mathrm{MHz}$ respectively for a 1.5 tesla MRI system.

It is evident that by the proper choice of frequency a specific nuclear species can be "tuned in" and observed in isolation. All commercial MRI systems operate at the hydrogen frequency with some capability to operate at other frequencies. Boron was not considered interesting and provisions for operation at the boron isotope frequencies were not included.

\section{Introduction}

The overall objective of this task is to develop a technology to noninvasively quantitate and localize boron in tirsue with magnetic resonance imaging and spectroscopy (MRI and MRS). Using boron MRS and chemical shift imaging (CSI), $11 \mathrm{~B}$ and $10 \mathrm{~B}$ concentration levels in tumors can be determined and followed over time.

Since present commercial MRI system manufacturers did not include magnetic resonance capability for boron, modifications to a system's hardware and soltware dependent on the boron relaxation times are required for obtaining spectroscopic and imaging information. Analysis software, preferably on an off line coinputer, is also necessary to understand and interpret/reconstruct this data.

This report describes the GE Signa ${ }^{\mathrm{TM}} 1.5$ Tesla MRI system hardware modifications required to obtain $M R$ information at the boron frequencies, the software required to image and analyze the resultant data, and the operation of the system while performing boron MRS and CSI. 


\section{Hardware Requirements}

The major subsystems of the GE Signa ${ }^{T M}$ MRI system (Figure 1) were analyzed for frequency and software dependencies. In some cases, the effect is minor and does not merit modification. In other cases, the subsystem requires replacement or modification. quencies within that range. These frequencies do not include ${ }^{10} \mathrm{~B}$ operation at $6.86 \mathrm{MHz}$ and ${ }^{11} \mathrm{~B}$ at 20.49 $\mathrm{MHz}$. Since the present RF power amplifier can not be modified (present capability must be preserved), the only option is another RF amplifier capable of operation at the boron frequencies which can bypass the present Signa ${ }^{T M}$ RF amplifier.

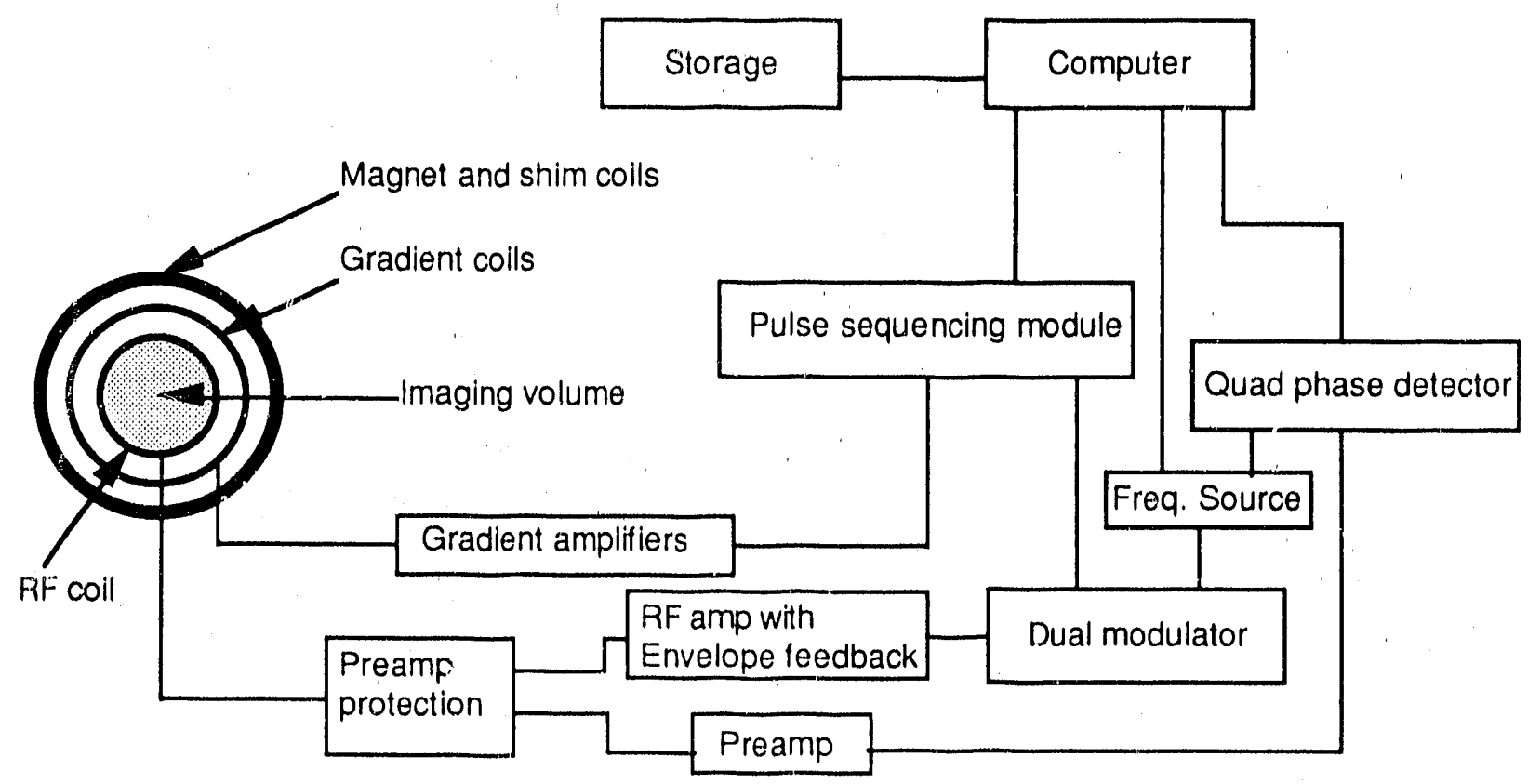

Figure 1. MRI subsystems for the GE Signa ${ }^{T M}$ MRI system. Only those systems affected by boron operation are indicated.

With the requirement of modification or replacement of subsystems to operate at a different frequency, it should be remembered that the MRI system must not have its normal patient scanning at the hydrogen frequency compromised. Going from hydrogen (proton) operation to boron or from boron to proton operation must not require more than one hour. Therefore, with frequency sensitivity and the changing of operation as requirements, the following modules or subsystems require modification:

RF Power Amplifier. The frequency range of operation of the GE Signa ${ }^{\mathrm{TM}} \mathrm{RF}$ power amplificr is 10 to $80 \mathrm{MHz}$. However, this amplifier has the capability of operating at only seven discrete fre-
Preamplifier. The frequency range of the preamplifier is narrow band, limited to operation at the proton frequency of $63.86 \mathrm{MHz}$. Off-frequency operation would decrease the already critical signal-tonoise and overall gain. Since the boron isotopes have a much lower NMR sensitivity than proton, signal-tonoise becomes significantly more important. Modification of a proton preamplifier for $11 \mathrm{~B}$ frequency is straight forward, requiring only an adjustment of the impedarice matching network to the input of the first amplifier stage at the operating frequency. Because of the preamplifier's small size and portability, it can be replaced when operating at either boron frequency,

Preamplifier protection. Since the voltagc 
sensitive preamplifier is connected to the same RF coil as the RF power amplifier, a protection network must be used when the RF power amplifier is transmitting. This network must withstand a maximum power of 20,000 watts. This is accomplished with frequency sensitive impedance transformers which can standoff the high voltage and currents developed within this system.

Quarter wavelength impedance transformers $(\lambda / 4$ transmission cables) with shorted outputs transform this short to an open at their inputs. Shorting these transmission cable sections is accomplished by passin : a positive steady state current pulse through PIN diodes during the transmit phase. Figure 2 shows how this nelwork is connecled.

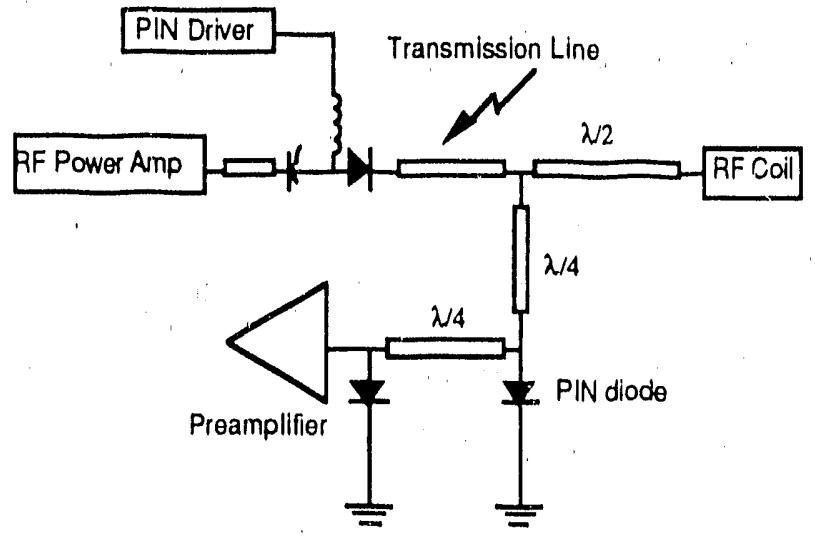

Figure 2. Transmit/Reccive switching.

During the receiving phase the signal from the RF coil is blocked from entering the RF power amplifier by a PIN diode which has been back biased (negative applied voltage) and is conducted to the preamplifier through a full wavelength transmission cable. The PIN diodes which were used to short the $\lambda / 4$ transmission sections are turned off and "disappear" within the protection network.

At $63.86 \mathrm{MHz}$, the $\lambda / 4$ transmission lines are about 30 inches. However, this length increases to about 100 inches for $20.49 \mathrm{MHz}$ and to almost 300 inches for $6.86 \mathrm{MHz}$. Instead of using transmission lines, the same impedance networks can be formed with lumped elements, i.e., discrete capacitors and inductors. A new network can be designed which will bypass the present network for proton operation.
RF colls. Not only must the active subsystems (RF power amplifier and preamplifier) be tuned to the boron frequencies, but the RF coils which apply a magnetic field in the transverse plane must be designed for the same frequency of operation. The coils provided with the Signa ${ }^{\text {TM }}$ system are tuned only to the proton frequency and are narrow band (high $Q$ ), disallowing operation at any other frequency. In addition, coils are designed for specific applications which adapt to a particular anatomy (head, body or surface). This passive subsystem can be quickly changed ty replacement.

Envelope Feedback. This module samples the envelope of the RF power amplifier output and fecdbacks a gain correction to the input, if needed, maintaining a constant RF output. It offers wide band operation from 10 to $80 \mathrm{MHz}$ but would need modification at $6.86 \mathrm{MHz}$. Unless it is found that the boron applications require the additional stability, the envelope feedback will not be used.

Frequency Source. The frequency sources for transmitter and receiver operation are provided by two frequency synthesizers made by PTS. Frequency selection is under software control but is inhibited for operation at frequencies not supported by the Signa ${ }^{\text {TM }}$ RF power amplifier. The frequency synthesizers can be manually controlled, but loop back is required to satisfy operating conditions expected by the Signa ${ }^{\text {TM }}$ computer. This can be accomplished by replacing of the frequency synthesizers' connector with a loopback connector.

Gradient Amplifler. Not only is the magnetogyric ratio innportant in determining the bulk magnetization and, therefore, the Larmor frequency, but for imaging it is important in determining the position of the originating NMR signals and, consequently, the resolution of the imaging sequence. Larmor's equation can be expanded to include the effect of gradient magnetization. This effect is cmall compared to the main static field (15,000 gauss vs. I gauss $/ \mathrm{cm}$ ) but is large enough to yicld resolution in the millimeters.

Equation (2) shows how the Larmor frequency (I) is altered in the presence of the homogeneous $B_{0}$ field by the addition of a liitear ficld gradient, $G_{x}$, along the $X$ axis $\left(G_{y}\right.$ and $G_{z}$ add in a similar way). These 
gradients can also be time varying as well as static. The fields produced by these gradients are parallel to the main $B_{0}$ field but vary in the $X, Y$, and $Z$ planes.

$\left[=\gamma\left(B_{0}+x G_{x}\right)\right.$

Since the $\gamma^{\prime}$ s of ${ }^{11} \mathrm{~B}$ and ${ }^{10} \mathrm{~B}$ are less than that of hydrogen $\left({ }^{1} \mathrm{H}\right)$, the gradient current required for boron nuclei magnetization must be increased to obtain similar resolution. The base design of the gradient amplifiers and coils has been optimized for hydrogen. The nuclei magnetization, which is proportional to current output, can not be increased without adding more amplifiers and/or by changing the gradient coil design. Changing the amplifiers' configuration is not an option since normal hydrogen imaging must bc maintained at the site where the modifications are made.

In addition, experiments on a GE CSI-II Imager/Spectrometer have shown the relaxation times in tissue for the boron compound $\left(\mathrm{Na}_{2} \mathrm{~B}_{12} \mathrm{H}_{11} \mathrm{SH}\right.$ borocaptate sodium or $\mathrm{BSH}$ ) to be much shorter than for hydrogen, requiring a faster "playout" of the gradient pulses. One of the relaxation time parameters, $\Gamma 2$, is less than $1 \mathrm{msec}$ for ${ }^{11} \mathrm{~B}$ in tissue. This means the signal coming from the nuclei decreases exponentially with a time constant of less than one msec. This is an important factor when considering the rise or fall times of the gradient output which are about $600 \mu \mathrm{sec}$ from zero to fullscale or fullscale to zero. Given these constraints, programs which control the pulsing of the gradients must "playout" the magnetization pulses in a shorter time period in order to acquire the fast decaying signals from the boron nuclei.

With the restrictions mentioned, the only hardware options are to work with the system as is accepting a lower resolution or design smaller gradient coils which can be easily installed without modifying existing coils and cabling. At this point the system will be maintained without change and only the scanning software will change, compensating for some hardware deficiencies and the faster relaxation time parameters.

Quad Phase Detector (QPD). To avoid the display of signals folded around the frequency of operation, detection of the RF is performed in quadrature (Figure 3). Proper operation depends on the two phase-sensitive detectors, PSD, having very similar phase and gain. Imperfect gain and phase adjustments lead to ghosts and glitches, i.c., unwanted phantom signals folded in from some other region of the spectrum.

The phase matching for the QPD is designed for operation from $25 \mathrm{MHz}$ to $64 \mathrm{MHz}$ and, therefore, additional phase compensation for lower frequencies may be required in the phase sensitive detector. This phase compensation is achieved by adding more capacitance in each detector. This would lower the upper frequency limit of the modules possibly climinating $63.86 \mathrm{MHz}$ operation. Since operation at $63.86 \mathrm{MHz}$ must be maintained, a module which is removable will be modified and used if operating at the lower frequencies produces the ghosting artifacts.

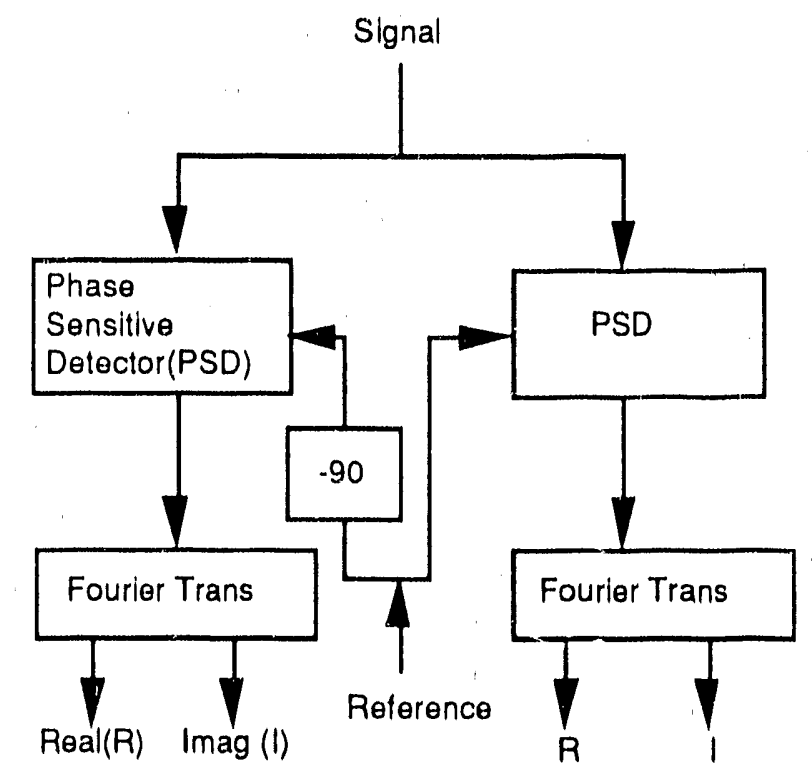

Figure 3. Quadrature Phase Detection

Dual Modulator. The dual modulator provides the necessary modulated RF input to the RF amplifier and has similar operational concerns as the QPD. A modified dual modulator can be made available if operation merits replacement of the existing dual modulator.

The rest of the subsystems shown in Figure 1 will not require modification and can be used as configured. 


\section{Description of Hardware Modifications}

\section{RF Power Amplifier}

Using the specification described in Appendix A, Kalmus Enginecring of Woodinville, Wa., designed an RF power amplifier to be used in place of the present Signa ${ }^{\mathrm{TM}} \mathrm{RF}$ power amplifier. There were only two changes to the specification: (1) lincarity to within $\pm 2 \mathrm{~dB}$ instead of $\pm 1 \mathrm{~dB}$, and (2) harmonics to be less than $-20 \mathrm{~dB}$ instead of $-30 \mathrm{~dB}$. To mect the power supply droop, a higher power amplifier (4000 watts instead of 2000 watts) was required. The amplifier system meeting all the specifications was a Kalmus model 169LF \& HI-Pulsed Amplificr modified for a frequency range of 6-30 $\mathrm{MHz}$ (Figure 4).

The Model 169LPL is a wideband pulse amplifier which can instantancously cover $6-30 \mathrm{MHz}$ at a power output level up to 4000 Watts (W) into a $50 \Omega$ load. The overall system gain is $63 \mathrm{~dB}$ giving 2000 W output for $1 \mathrm{~mW}$ input. Final test results for the Kalmus model 169LPL Amplifier are included in Appendix. B with an operating manual. When operating at $6.86 \mathrm{MHz}$ for ${ }^{10} \mathrm{~B}$, a $6-12 \mathrm{MHz}$ low pass filter must be connected to the output to eliminate harmonics from 13 to $30 \mathrm{MHZ}$.

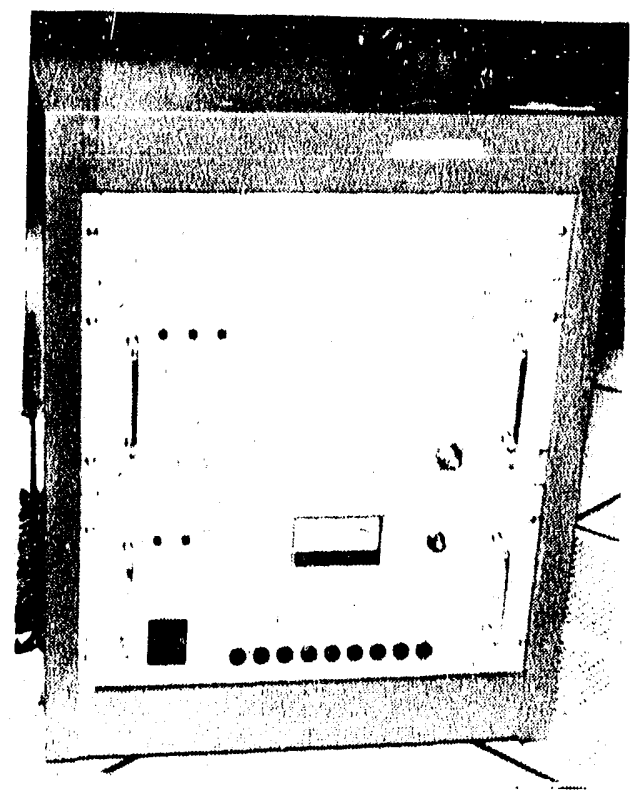

Figure 4. Boron RF power amplifier
The "boron" RF power amplifier must be located close to the Power Distribution Unit (PDU) of the MRI system so power can be provided via a spare gradient amplifier circuit breake. The amplifier is hardwired to this circuit breaker permitting power control from the on/off switch on the front panel of the RF amplifier.

The "boron" RF power amplifier requires a low level signal input and an "unblank" signal to gate the power output. The low-level signal input is provided by the dual modulator which has a maximum output of $0 \mathrm{dBm}$ (for 50 ohms system, $0 \mathrm{dBm}=1 \mathrm{~mW}$ ). The dual modulator output is accessed from a BNC connector marked "atten" (atteriuation) on the patch pancl (Figure 5), situated above the Service Control Module (SCM) circuit boards. The short cable connecting the "atten" to "RF Amp" must be disconnected and a longer cable from "atten" is connected to the Kalmus amplificr.

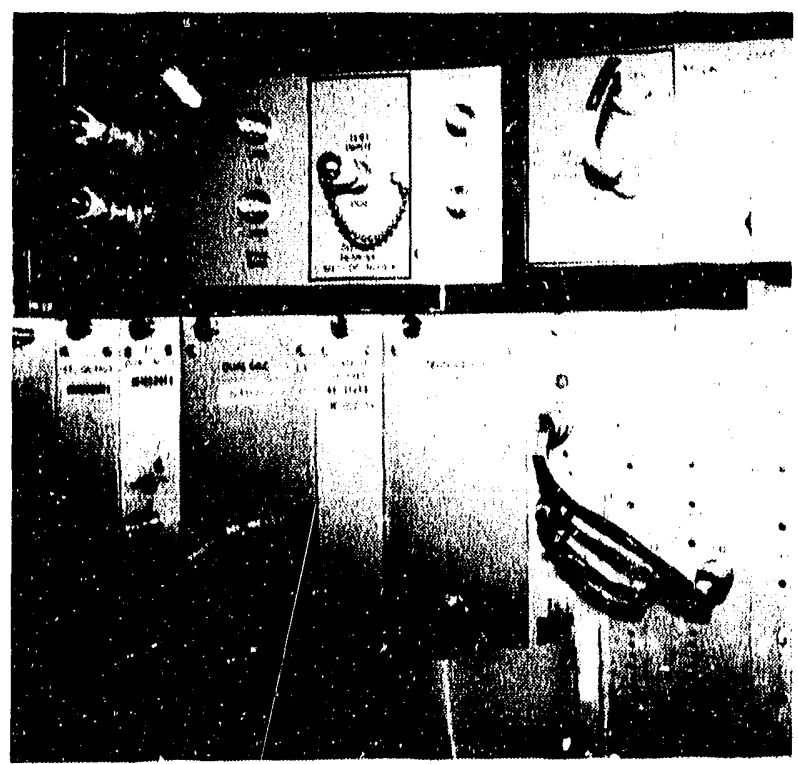

Figure 5. Front view of the patch panel

Since most boron imaging applications require less than $1500 \mathrm{~W}$, the power output can be changed via software controllable attenuators in the dual modulator, before signal output at the "atten" BNC connector. These attenuators can be selected at the time of scanning from the operator's console and provide a maximum attenuation of $23.9 \mathrm{~dB}$ in $0.1 \mathrm{~dB}$ steps. Since the surface coil applications need even 
less power than imaging sequences, an additional 20 $\mathrm{dB}$ in attenuation must be added to the Kalmus RF amplifier input.

"Unblank" to the amplifier is also obtained from the patch panel, seen in Figure 5. "Unblank" is a control signal which gates RF coincident with the length of "unblank". Because the polarity specification for the RF amplifier's "unblank" input was opposite of what was provided by Signa ${ }^{\mathrm{TM}}$, a circuit powered by a nine volt battery was designed to invert the signal from the "unblank" on the patch panel before reaching the RF amplifier. This circuit is depicted in Figure 6.

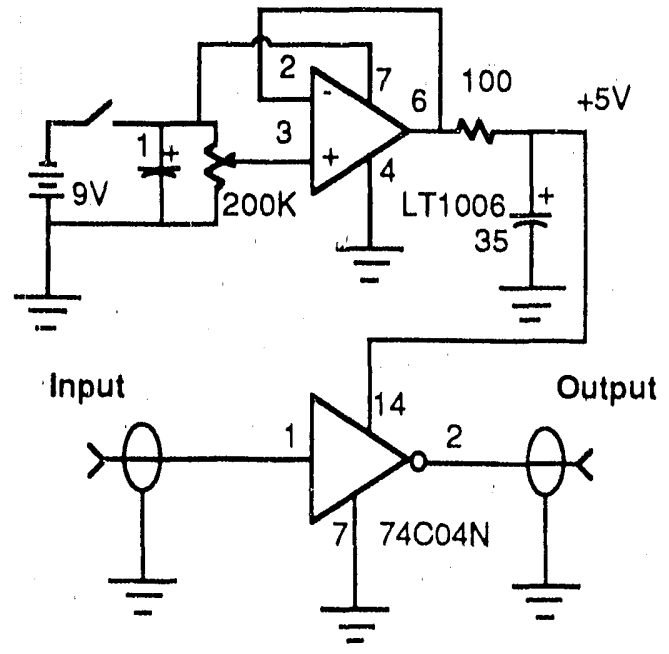

Figure 6. Inverter for "Unblank" signal from patch pancl. Output goes to RF amp.

The Signa ${ }^{\mathrm{TM}}$ RF power amplifier (ETO amplifier) has two outputs: one for bodies and one for heads. The original output to the head port is disconnected from the envelope feedback panel and connected to a 50 ohms $(\Omega)$ dummy load. The output of the Kalmus amplifier is then connected to the head port (Figure 7) with either an RG-9/4/ or RG-214/U cable terminated with type $\mathrm{N}$ connectors.

\section{Preamplifier}

The proton preamplifier has a gain of $40 \mathrm{~dB}$ and a noise figure of about $1.1 \mathrm{~dB}$. The input impedance is matched to $50 \mathrm{ohms}$ at $63.86 \mathrm{MHz}$. The matching network on the input of the preamplifier is narrow, reducing the noise (improving the noise figure) and providing a source impedance for the input transistor for optimum noise performance. The source impedance to the input of the preamp is a combination of a loaded RF coil seen after 4-quarter wavelength transformers (sec Figure 2).

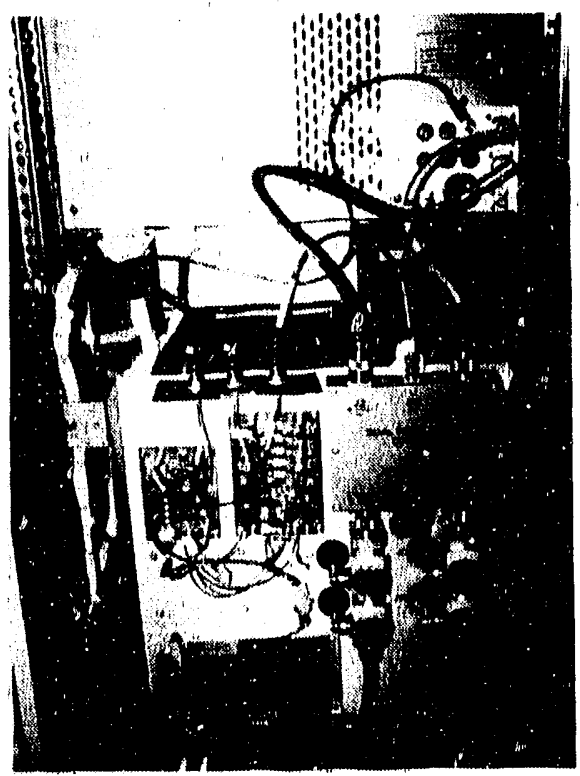

Figure 7. Back view of the RF cabinet showing coil ports.

Before the preamplifier's front end (Figure 8) can be modified for the two different boron isotope frequencies the input impedance must be characterized. The input impedance of the first stage transistor was 195 - j177 ohms at $6.86 \mathrm{MHz}$ and 77.3 - j83.5 ohms at $20.49 \mathrm{MHz}$. Using equations (3) and (4) the series impedances, $R_{s}$ (series resistance) and $X_{s}$ (series actance or imaginary part of the impedance) of the first stage of the preamplifier can be translated to parallel impedances, $R_{p}$ and $X_{p} .^{2}$

$\mathrm{R}_{\mathrm{p}}=\mathrm{R}_{\mathrm{s}}\left(1+(\mathrm{Q})^{2}\right)$

$X_{p}=\frac{R_{p}}{Q}$

where $Q=\frac{X_{5}}{R_{6}}$.

With parallel impedances, an equivalent circuit is 
developed which can be matched to $50 \Omega$ using an $\mathrm{L}$-section configuration (Figure 8 ). The inductive reactance, $X_{L}$, and capacitive reactance, $X_{C P}$ can be found with the following equations:

$$
\begin{aligned}
& X_{L}=\sqrt{50 R_{P}-50^{2}} \\
& X_{C P}=-\left(\frac{50 R_{P}}{X_{L}}\right)
\end{aligned}
$$

$X_{C P}$ is a combination of $C_{11}$ and $C_{p}$ and $X_{L}$ is the impedance of $L_{10}$. The series to parallel transformation gives $R_{p}=466 \Omega$ and $X_{p}=-215 \Omega$ for 6.86 $\mathrm{MHz}$ and $\mathrm{R}_{\mathrm{p}}=168 \Omega$ and $\mathrm{X}_{\mathrm{p}}=-155 \Omega$ for 20.49 $\mathrm{MHz}$. The resultant inductor, $\mathrm{L}_{10}$, and capacitor, $\mathrm{C}_{11}$ for $6.86 \mathrm{MHz}$ and $20.49 \mathrm{MHz}$, respectively, are $3.3 \mu \mathrm{H}$ and $35 \mathrm{pF}$ for $6.86 \mathrm{MH} / \mathrm{Z}$ and $.53 \mu \mathrm{H}$ and 13 $\mathrm{pF}$ for $20.49 \mathrm{MHz}$.

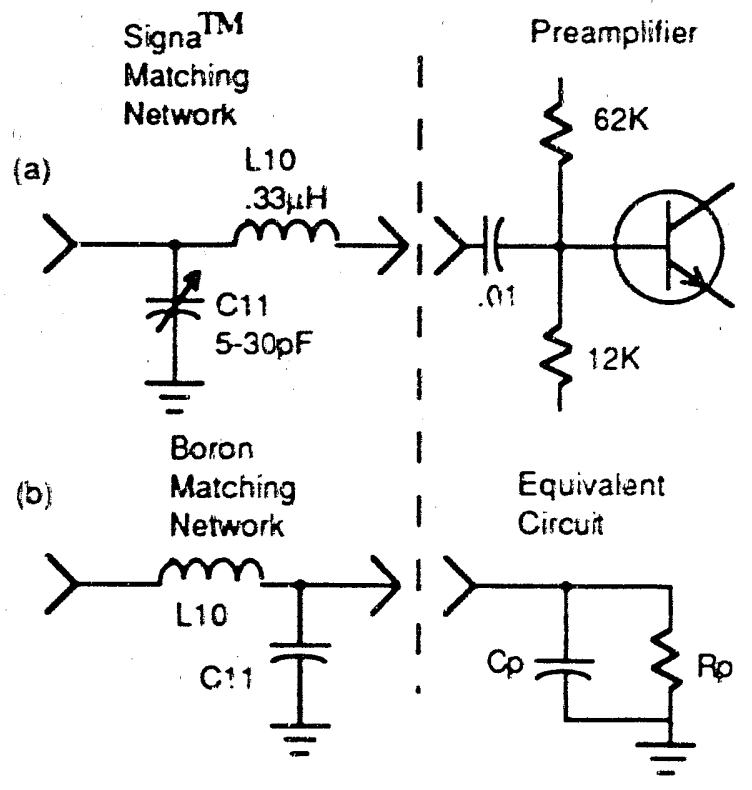

Figure 8. Front end of the MRI preamplifier. (a) proton matching network and first stage of the preamp circuit. (b) equivalent circuit of the preamp front end and the matching L-section for boron frequencies.

After modification, the gain of each preamplifier was measured as $62 \mathrm{~dB}$ at $6.86 \mathrm{MHz}$ and $55 \mathrm{~dB}$ at $20.5 \mathrm{MHz}$ with a noise figure of $1.0 \mathrm{~dB}$. (Noise ligure is an index which compares input signal-toroise, $S \wedge$, to output $S \wedge$. The lower the better.) $A$ noise figure was not obtained for $6.86 \mathrm{MHz}$, as the noise figure meter (HP-8970B) does not operate below $10 \mathrm{MHz}$.

\section{Preamplifier Protection}

For preamplfier protection, the Signa ${ }^{\text {TM }}$ system uses quarter wavelength transmission lines nested within the patient table support located at the back end of the superconducting magner. Since the present hydrogen (proton) coils and preamplifier protection are not needed for the boron studies, they will be left in place, disconnected from the RF power amplifier and receiver. The boron preamplifier and preamplifier protection will be connected extemally to the patient support table bypassing the present system.

Al the boron isotope frequencies, quarter wavelength transinission lines become lengthy and cumbersome. The same effect can be achieved using a lumped element network (discrete inductors and capacitors) 60 reduce the size (Figure 9).

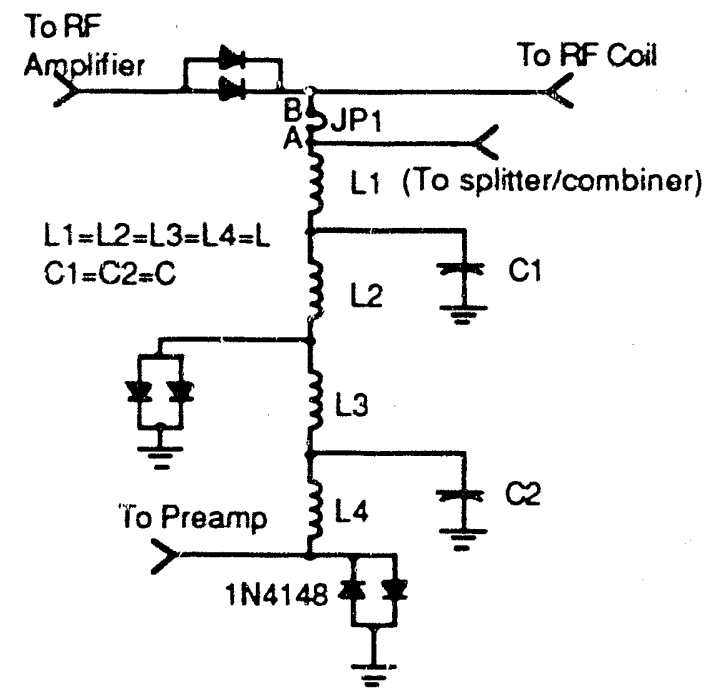

Figure 9. Transmil/ Receive protection circuitry for preamplifier for linear coils. All diodes are PIN unless otherwise specified.

With JP1 connected, the circuit in Figure 9 will protect preamplifiers with the same frequency of design as the protection circuitry but only linear volume or surface coils (as opposed to quadrature coils) may be connected to this protective circuitry. 
With JP1 disconnected, this circuit can be connected to quadrature coils through a "splitter/combiner". Quadrature coils and associated splitter/combiner techniques will be explained in the next section, RF coils.

The T-networks of the protection circuitry are actually quarter wavelength transmission sections. The components of each T-network are determined using equations (7) and (8) at the frequency of operation (for a $50 \Omega$ system). A T-network is formed by an inductor-capacitor-inductor (L-C-L) combination. $L_{1}-C_{1}-L_{2}$ and $L_{3}-C_{2}-L_{4}$ are separate $T$. networks.

$\mathrm{L}=\frac{50}{2 \pi \mathrm{f}_{0}}(\mathrm{H})$
$\mathrm{C}=\frac{1}{2 \pi \mathrm{f}_{0} 50}$

When the PIN diodes are biased with sufficient current during the transmit mode, the quarter wavelengths are shorted, forcing a high impedance condition at the opposite end and effectively blocking high power from flowing to the preamplifier. Back biasing the PIN diodes during the receive mode shuts off the direct current (DC) nowing through the circuit and configures the $T$-networks as standard transmission lines with a characteristic impedance of $50 \Omega$. Since RF power will have been sufficiently reduced after the first quarter wavelength section, regular diodes (1N4148) may be used for the second $T$ network. The low level NMR signal coming from the coil during the receive mode will not have sufficient voltage to turn on any of the diodes maintaining the $50 \Omega$ characteristic impedance of the network.

\section{RF Coils}

Background. RF coils for MR have two primary functions: excitation of nuclear spins and detection of the resulting precession. As a "transducer", efficiency is a primary concern. This corresponds to generating a maximum $B_{1}$ magnetic field (transverse to the $B_{0}$ main magnetic field) within the saruple volume during the transmission mode, and receiving with a minimal degradation of signal-to-noise ratio of the sample volume during the reception mode. To ensurc linear response within a sample volume the $B_{1}$ field must be spatially uniform (homogeneous). The two conditions of efficiency and spatial uniformity can not always be met, requiring compromise in MR coil design.

Another way to view an RF coil is as a magnetic storage device. As a magnetic storage device, radiation energy is kept in the near field temporarily and does not radiate to the far field. In the transmission mode, spinning nuclei will absorb some of this energy in the near field. In the reception mode, this device must also detect the magnetic field of spinning nuclei without extracting too much energy. Extracting too much energy will result in a shortening of the NMR signal. Resonant "LC" circuits (inductor-capacitor tank circuits) are reasonable energy storage devices and are used for all MR coil designs. ${ }^{3}$ In this discussion three types of RF coils for MR will be discussed: surface, linear volume, and quadrature.

Before any coil can be discussed, several parameters upon which a coil's design is based will be defined. Of primary concern are the coil $\mathrm{Q}$, a measure of the circuit's energy storage efficiency, and the coil's filling factor, $\eta$, defined as the ratio of RF magnetic energy stored in the sample volume to the total RF magnetic energy stored in the coil. This means that the more material enclosed within the coil, the higher will be the filling factor.

The $\mathrm{Q}$ of a coil can be determined by either measuring the center frequency, $f_{c}$, and dividing by the bandwidth, $f_{2}-f_{1}(\Delta f)$, where $f_{2}$ and $f_{1}$ are the $-3 d B$ points, or measuring the inductance, $L$, and the series resistance, $r_{s}$, and calculating $Q$ from equation (9). It is usually easier to do the frequency measurements. Note that $Q$ is inversely proportional to the resistance of the coil, $r_{\text {coil }}$. This resistance is greater than the DC resistance of the coil's conductor, since it is proportional to the square root of the frequericy due to the RF skin effect. Therefore, the higher the frequency, the greater will be the coil's resistance due to the RF skin effect.

$Q=\frac{f_{c}}{\Delta f}=\frac{\omega L}{r_{s}}$ 
For imaging purposes, Hoult and Lauterbur ${ }^{4}$ demonstrated there are other noise contributing losses, decreasing the observed $Q$ of a coil. These losses are due to magnetically :nduced eddy currents and dielectric or conductive losses due to stray electric fields in the sample. They pointed out that magnetically induced sample losses could become the dominant noise source. These losses can be defiried as a coupled sample resistance, $r_{s a m p l e}$. $r_{s}$ now becomes a combination of $r_{\text {sample }}$ and $r_{\text {coil }}$. With this in mind there are two coil Q's to be considered: $\mathrm{Q}_{\text {empty }}$ and $\mathrm{Q}_{\text {loaded. }} \mathrm{Q}_{\text {loaded }}$ is measured with a sample in the coil. This will be a lower $\mathrm{Q}$ due to the coupled resistance from the sample. This $Q$ is the one used in most calculations. Obviously, the coil without a sample will result in the cmpty $Q$ $\left(Q_{\text {empty }}\right)$. The best indicator of coil sensitivity ${ }^{3}$ neglecting any inductance changes is the ratio

$$
\frac{Q_{\text {anply }}}{Q_{\text {loided }}}=\frac{r_{\text {coil }}+r_{\text {sample }}}{r_{\text {coil }}} \text {. }
$$

One of the effects of having a conducting medium for a sample is the resultant electric ficlds that are induced. This has the added effect of reducing the apparent inductance of the coil and thus raising the frequency of coil resonance. The overall effect makes calculation of the coil's resonance difficult. One can only expect to be somewhere in the "ballpark". The final configuration of an RF coil must be tuned with an expected load (dog head) and in the operating location within the bore of the magnet.

Since dissipative material can raise the series resistance of a coil lowering the $Q$, care must be taken in the selection of the material for the coil design. A high $\mathrm{Q}$ means there will be a high current flowing in the coil producing the highest possible RF $B_{1}$ field.

Surface Colls. Surface coils may be thought of as short solenoids with about half of the magnetic energy in the sample. This factor produces a large filling factor and couples strongly to the sample. But the $B_{1}$ field and the corresponding signal sensitivities are highly inhomogeneous. At the price of increased signal-to-noise there is a great loss in signal strength uniformity within the sample. Along the axis of a surface coil, $X$ direction, the field per unit current varies as

$$
B_{1} \rightarrow \frac{1}{a\left(1+\frac{x^{2}}{a^{2}}\right)^{\frac{3}{2}}}
$$

The signal sensitivity $B_{1}$ at the center of the coil $(x=0)$ is inversely proportional to the coil radius, a (See Reference 3). The effectiveness of a surface coil is quickly lost with increasing depth into a sample. Surface coils having large filling factors are still effective, but, for boron imaging applications, the volume coils produce better results.

A surface coil, three inches in diameter with four turns of No. 12 wire, resulted in a loaded $Q$ of about 60. This coil is shown schematically in Figure 10.

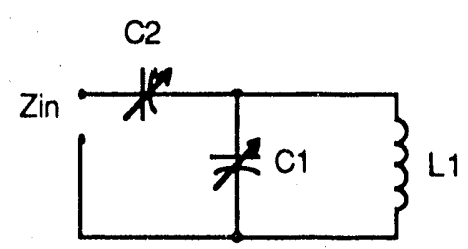

Flgure 10. Schematic of a typical surface coil and matching circuit.

The surface coil is resonated by adjusting $C_{1}$, and the input impedance (must be matched to $50 \Omega$ ) is varied by adjusting $C_{2}$. Since changing $C_{2}$ affects the resonance, an iterative process between $C_{1}$ and $C_{2}$ is required before the input impedance and the frequency of resonance are sct. The fixed capacitors were ultrahigh $Q$ porcelain capacitors from Dielectric Laboratories, Inc and the variable capacitors were high $Q$, tubular capacitors with PTFE fluorocarbon diclectric from Oxley. By definition, all components must be nonmagnetic to eliminate RF inhomogeneity from magnetic field distortion around ferromagnetic materials.

Surface cuils made from No. 12 wire resulted in good $Q$, but les:s than that developed in a volume coil made from either solid copper or low-loss, flexible circuit boa-d. The lower $\mathrm{Q}$ is due primarily to the increased resistance from the wire's skin effect. The surface coils made of low loss, flexible circuit board (UltraLam from Rogers Corporation) with a teflon diclectric, 4 mils thick, between 1 oz copper clad, 
exhibited a t uch higher Q. With a dielectric constant of 2.40 th material can also be used for very low loss capacitors of about $135 \mathrm{pF} / \mathrm{in}^{2}$.

Bringing a coil in close approximation to tissue or other conducting medium not only loads the coil, reducing $Q$, but reduces coil inductance. This requires tuning the coil in close contact with the sample to be analyzed. In addition, there are conducting structures within the magnet bore which reduce the coil's inductance slightly, requiring coil tuning at the proper location within the bore of the magnet.

Several different matching networks (circuitry to match the coil to $50 \Omega$ ) were developed, but there was not a demonstrable signal-to-noise advantage of one over the other. Distributing capacitance at symmetrical places around the loop in licu of a lumped sum at the terminal ends of the coil reduced large clectric ficld effects, thereby making the coil less susceptible to loading changes, but the value of each individual capacitor must be increased to compensate for putting them in series. Larger capacitors tend to have more dissipative losses.

Linear Volume Colls. Several types of volume coils were considered for the boron imaging applications. These include solenoid, slotied solenoid, and saddle. Each coil was evaluated on the basis of use, ease of construction and $B_{1} R F$ homogeneity.

Since the application planned for the volume coil included imaging small animals (rabbits), a slotted solenoid configuration, which could be aligned in the transverse direction, was selected. A small slotted solenoid constructed of a single sheet of copper has better RF homogeneity than a wire solenoid or saddle coil, since it channels the magnetic flux in one end of the cylinder to the other forming a "flux pipe". 5 In addition, this type of coil is relatively casy to construct. RF is coupled to the slotted solenoid inductively through another single turn coil aligned on the same axis. This has the advantage of keeping impedance matching independent of the luning.

The "inductively-coupled" linear volume coil used for imaging small animals was fabricated on an acrylic cylinder with a $10-\mathrm{cm}$ diameter and a $15.2-\mathrm{cm}$ length. This coil was constructed by wrapping a piece of copper foil $7.7 \mathrm{~cm}$ wide around the cylinder and placing a capacitor madc from the high $Q$ flexible circuit board material between the long edges of the foil. The driver coil, mace of $1.2 \mathrm{~cm}$ wide copper foil, was placed on the same cylinder as the main coil. Schematically this coil is shown in Figure 11. The main coil is tuned for ${ }^{11} \mathrm{~B}$ resonance by adjusting $\mathrm{C}_{2}$. Resonance of the driver coil is independently tuned with $C_{1}$. This adjustment is not as critical since the $Q$ of this circuit is very low. (The $Q$ is lowered by putting the transmission cable impedance, $50 \Omega$, in serics with the driver coil.) Each capacitor, $\mathrm{C}_{1}$ and $\mathrm{C}_{2}$, is a combination of flexible circuit board, cut for a particular capacitance, and a variable tubular capacitor.

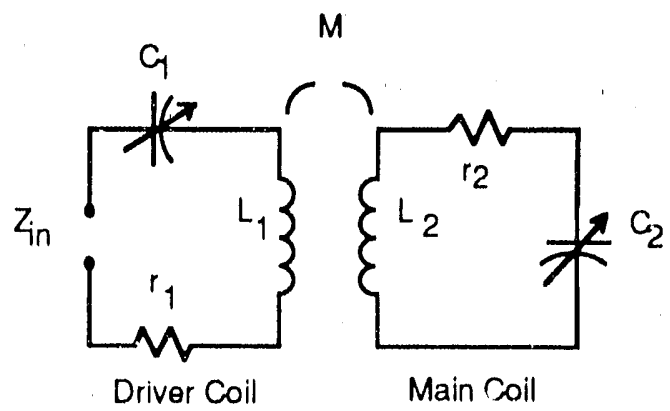

Figure 11. Schematic of a volume coil using inductive drive.

The volume coil shown in Figure 11 is explained mathematically in the following manner ${ }^{6}$ :

$Z_{i n}=r_{1}+j\left(\omega L_{1}-\frac{1}{\omega C_{1}}\right)+\frac{(\omega M)^{2}}{r_{2}+j\left(\omega L_{2}-\frac{1}{\omega C_{2}}\right)}$

$\mathrm{Z}_{\mathrm{in}}=\mathrm{r}_{1}+\frac{(\omega \mathrm{M})^{2}}{\mathrm{r}_{2}} \quad$ (at resonance)

$\mathrm{Z}_{\text {in }} \approx \frac{(\omega M)^{2}}{r_{2}}$

where $\mathrm{M}=\mathrm{k} \sqrt{\mathrm{L}_{1} \mathrm{~L}_{2}}$

and $\omega=\frac{1}{\sqrt{L_{1} C_{1}}}=\frac{1}{\sqrt{L_{2} C_{2}}}$.

When the two circuits are coupled through the inductors, $L_{1}$ and $L_{2}$, an additional inductance will 
result in each circuit called mutual inductance, $M$. At resonance, the inductive and capacitive reactance cancel leaving real resistances. As a result, the input impedance of the volume coil at resonance, $Z_{i n}$, becomes a combination of $(w M)^{2} / r_{2}$ and $r_{1}$. Because $r_{1}$ $\ll$ than $(w M)^{2} / r_{2}$, the input impedance $Z_{\text {in }}$ becomes $(w M)^{2} / r_{2}$. Given $M$ and $r_{2}$ are of sufficient quantity and both coils are tuned to resonance, the input impedance can be set by adjusting the distance between the driver coil and the main coil, since the coupling constant, $k$, and therefore, $M$, is dependent on spacing.

Connection to either the surface coil or linear volume coil is through an RG-58A/U coax cable cut to a length of $17 \mathrm{fect}$ and then trimmed using an impedance meter to form a half wave transmission line. Theoretically, this length is about $16.75 \mathrm{fect}$ for $20.49 \mathrm{MHz}$.

Quadrature Volume Colls. Although the surface and linear volume coils are useful for spectroscopy applications, the most versatile coil for boron imaging and spectroscopy is the quadrature volume coil. This coil decreases RF power requirement by a factor of 2 and increases $S / N$ by a factor of 1.414 over similar sized surface or linear coils. ${ }^{7}$ Since there is only one transmitter in the MRI system and the quadrature coil has two inputs, a splitter must be used to provide drive to the coil. To have a quadrature structure there must be at least 2 coils 90 degrees out of phase within the saine $\mathrm{Z}$ plane. In other words, if one coil is oriented in the $X-Z$ plane, the other coil must be in the $Y-Z$ plane. It is not essential that these coils be specifically aligned with the $X$ and $Y$ planes, only that they be 90 degrees out of phase. To increase the RF homogencity, more elements can be added in a symmetrical fashion, cqually spaced. An illustration of the basic geometry of this quad coil is shown in Figure 12.

The quadrature volume coil shown in Figure 12 has been called a "birdcage coil" 8 , where the wires are uniformly spaced in the azimuthal angle. Placement of capacitors along the straight segments configures this coil as a "low pass" type. Placing the capacitors uniformly around each end ring between the straight segments would configure the coil as a "high pass" type.

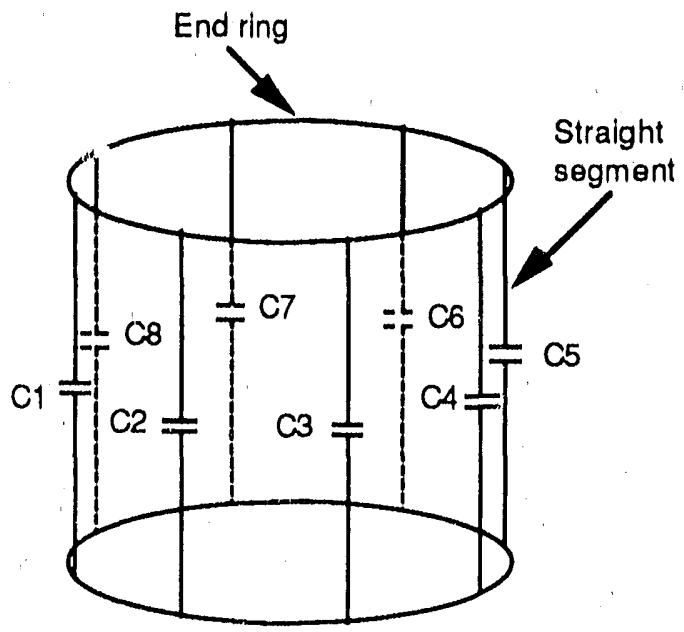

Figure 12. Illustration of the basic geometry of a low pass birdcage coil.

The coil is tuned for a single frequency by luning each segment and its mirror image segment. There are actually four coils in the birdcage zoil (Figure 12). Each coil consists of opposing long "wire" segments connected through the end rings with capacitors evenly spaced on the long segments.

The birdcage coil constructed for ${ }^{11} \mathrm{~B}$ imaging consists of 16 wire elements (flat copper strips) and capacitors in a low pass configuration resulting in eight coils within the quadrature structure. Increasing the number of elements increases the desired RF homogeneity.

The birdcage coil can be driven inductively with two circular driver coils placed 90 degrees apart. In this case, the axis of each driver coil aligns with a coil within the birdcage and energy is coupled into the coil that is parallel and, to a lesser degree, to the other coils. The standing waves which result in these coils produce a circularly polarized field which rotates at the same frequency of precession as the nuclei. Because there are multiple current paths available in a birdcage coil, many resonances will result. Only one of these resonances produces the homogeneous transverse $\mathrm{B}_{1}$ ficld.

To determine the actual resonance of the coil the output of a network analyzer (HP-8753B) is connected to one of the inductive drives and a search coil, about 1.5 inches in diameter, is used to sample the RF field 
completing the return path to the network analyzer. Using the input reflection mode of the network analyzer, multiple resonances will be displayed as inverted peaks indicating minimum reflection points. In the transfer mode, input to outpul, and with the search coil inside the birdcage coil, the resonances will be displayed as peaks indicating maximum gain. These peaks will vary in amplitude as the scarch coil is moved around within the confines of the birdcage coil because of the difference in RF homogeneity produced. The peak that changes the least is the primary resonance and produces the desired $B_{1}$ ficld. For the low pass coil configuration the lowest displayed frequency of resonance is the most linear and homogeneous. While in the transfer mode the coil's $Q$ can also be determined using the method described by equation (9).

After the coil is first made, a splisting of the primary resonance peak due to capacitance mismatch in each element will be observed. This splitung can be distinguished as a slight shifting of resonance as the search coil is moved around within the confines of the birdcage coil. Adding variable capacitors to half the elements, $\left(\mathrm{C}_{1}, \mathrm{C}_{2}, \mathrm{C}_{3}, \mathrm{C}_{4}\right.$, for an eight element birdcage) and adjusting each capacitance while observing the field with the search coil, peak splitting can be eliminated. As was mentioned in the background section of RF coils, the coil with a saline solutu on load approximating the volume of a dog's head must be fine tuned within the magnet bore, or a similar electrical environment. Since the saline solution "phantom" is only an approximation, large variation in dog head sizes will require additional small tuning adjustments before any MR experimentation can be conducted.

The 16 element ${ }^{11} \mathrm{~B}$ birdcage coil has a diameter of 7 inches and a length of 7 inches. Each clement is made of two 0.5 -inch wide copper traces, separated by a 0.1-inch gap in the middle and connected to the copper end rings. A single isolated copper trace of similar width is placed directly beneath the these top traces and is separated by the dielectric material of the flexible circuit material. This geometry produces a distributed capacitance in line with the natural inductance of each trace, reducing stray capacitance effects and also reducing the need for high lump capacitance connectung the top split traces. This geometry produced a capacitance of about $101 \mathrm{pF}$ per element. Resonance at $20.49 \mathrm{MHz}$ was achieved by adding a discrete $39 \mathrm{pF}$ high $\mathrm{Q}$ porcelain capacitor and a 1-30 $\mathrm{pF}$ variable capacitor across the gap in each element. The $\mathrm{Q}_{\text {empty }} / \mathrm{Q}_{\text {loaded }}$ was about 4-5, indicating good coil sensitivity.

Since the birdcage is a quadrature coil, its inputs must be phased 90 degrees from each other. These outputs can be derived from the RF power amplifier by using passive power splitters which will also phase the outputs properly. The outputs of a passive power splitter will each be at least $-3 \mathrm{~dB}$ from the original input and can be phased as required.

Most 90-degree splitter designs provide an option of "reversing" this phasing when used to combine two signals which are 90 degrees out of phase (receive mode). The "outputs" now become "inputs," the received signals being combined in phase before amplification by the preamplifier. This power splitter now becomes a comviner. The type of hybrid power combiner/splitter can be connected to the preamplifier protection circuitry (Figure 9) to form single coil transmit and receive capability for quadrature coils. The ${ }^{11} \mathrm{~B}$ quadrature hybrid power combiner/splitter used for imaging is demonstrated schematically in Figure 13. The preamplifier protection in Figure 9 is used with JP1 disconnected and point B connected to the splitter.

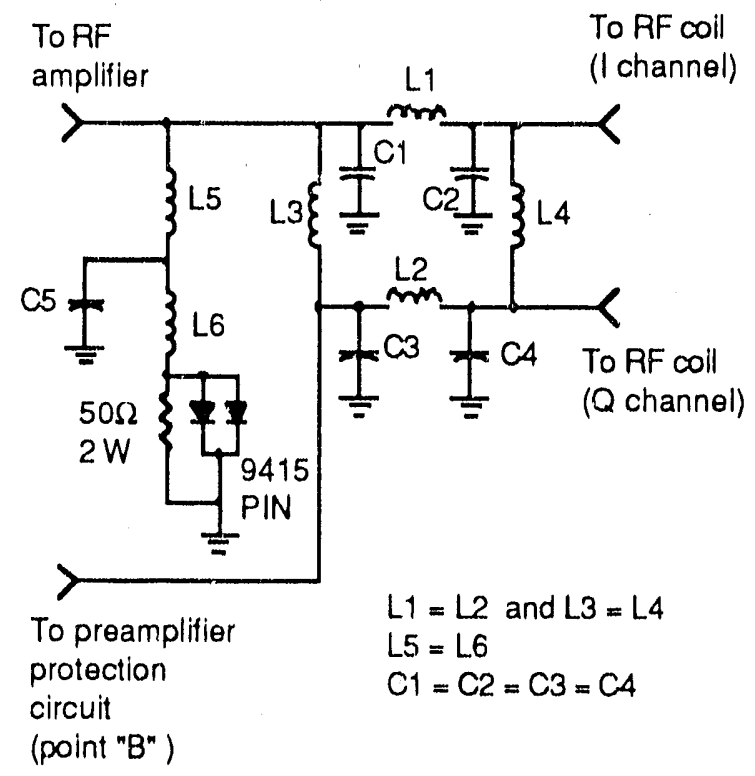

Figure 13. Quadrature hybrid power combiner/splitter for $50 \Omega 2 \mathrm{system}$. 
The T-network of $\mathrm{L}_{5}-\mathrm{C}_{5}-\mathrm{L}_{6}$ with the resistor and PIN diodes (Figure 10) form an attenuation pad which will absorb reflected power from the coil if any mismatch at the coil occurs. $\mathrm{L}_{5}, \mathrm{~L}_{6}$, and $\mathrm{C}_{5}$ are calculated using equations (7) and (8) as was done for the preamplifier protection circuitry. For an RF Amplifier and $\mathrm{RF}^{\prime}$ coil matched to $50 \Omega$, The other components, which form the quadrature combiner/splitter, are calculated using the following equations:

$\mathrm{L}_{1}=\frac{50}{2 \pi \mathrm{f}_{0}}$ and $\mathrm{L}_{3}=\mathrm{L}_{1} \sqrt{2}$

$C_{1}=\frac{1+\frac{1}{\sqrt{2}}}{2 \pi f_{0} 50}$

\section{Other Hardware Modifications}

No modifications were made on the envelope feedback assembly because actual operation did not indicate a need. Although changes were made to a QPD board, the resident QPD board was adequate for all spectroscopy and imaging sequences.

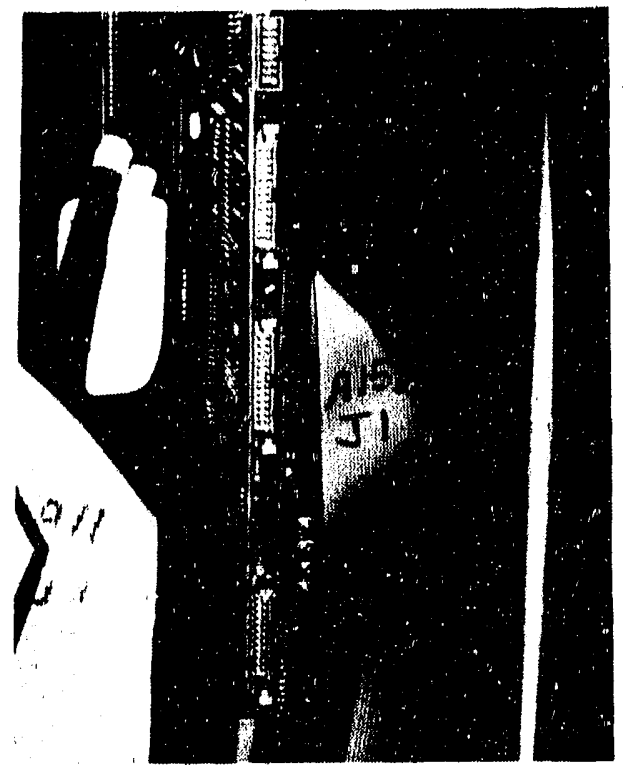

Figure 14. View of the wrap-around connector positioned on the SCM/PTS port.

For the frequency synthesizer, a terminating connector replaced the frequency synthesizer port cable located on the service control module (SCM). This connector feeds back the polling signals from the SCM to the SCM, satisfying software control requirements. This allows manual setting of the MRI transmit and receive frequencies different from that allowed from software. The position of this connector on the SCM board is shown in Figure 14. (The connector and the ribbon cable are numbered as A15$\mathrm{J} 1$ in the Figure.)

\section{Interfaces}

Macintosh Connection. Analyzing and displaying the data acquired from the Signa ${ }^{\mathrm{TM}}$ MRI system on the SignatM system requires an eifort beyond the scope of this project. However, software dovelopment using an off-line computer such as the Apple Macintosh II is more feasible and provides greater analysis flexibility. Additional hardwie and software are required to connect the Signa ${ }^{\mathrm{TM}}$ computer and download the raw data files to the Macintosh computer. Three different connecting options were considered.

1. A direct connection between the Macintosh RS-422 port (printer port) and the "con" channels on the IACK board of the Signa ${ }^{\text {TM }}$ computer (DG-4000 or DG-1708) can be made with shiclded twisted-pair cable. Con channels 6-13 may be used. The Macintosh computer is then set up as a terminal of the system.

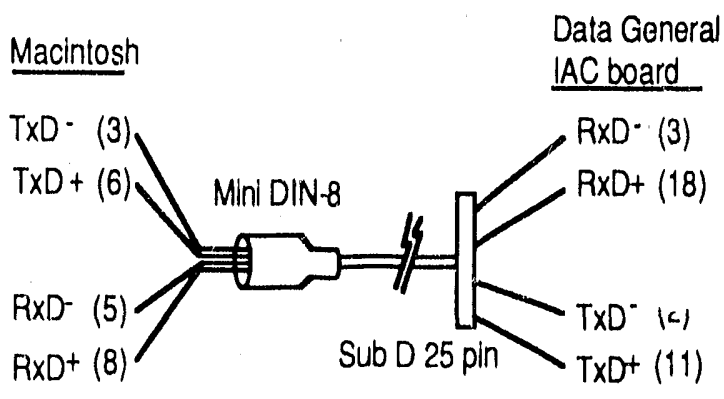

Figure 15. Macintosh to Data Gencral connection. TXD from one computer connects to RxD of the other computer maintaining the same polarity .

Using Versaterm software on the Macintosh, the Signa ${ }^{\mathrm{TM}}$ computer can be accessed and the data downloaded using "Kermit", a 
file transfer program, on both computers. Data transfer is limited to 197.00 baud rate. Figure 15 shows the cable betwe in these two computers and the pin-pin connection.

Figure 16 is a view of the back of the computer cabinet showing where the Macintosh cable connects to the iACK boards. These communication channels on the IACK boards are also known as "con" ports. This cable may be left in place without interferring with other Signa ${ }^{T M}$ functions.

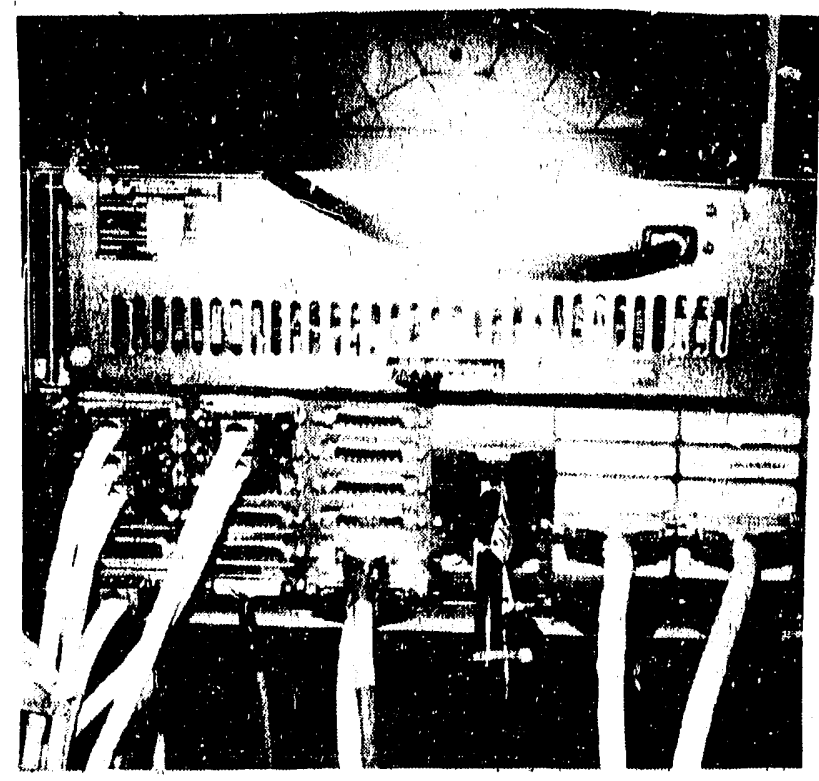

Figure 16. View of the IACK channels located in the rear of the computer cabinet.

2. Data can be written on magnetic tape by the Signa $^{\mathrm{TM}}$ computer and then transferred to the Macintosh from a compatible tape drive connected to the SCSI port of the Macintosh. This method is more expensive, but offers faster data transfer and is the best method of storing and later analyzing files of two megabytes or more.

3. Data can be transferred from the Signa ${ }^{T M}$ computer to the Macintosh via ethernet using the FTP protocol. Ethernet cards must be installed in both computers. Although this method is the most expensive, it offers the greatest flexibility and the fastest data transfer rate. 


\section{Software Development}

The software for this project requires writing programs for two different systems. Pulse programs for acquiring data are written for the GE. Signa ${ }^{T M} M R$ system in a pecific language known as pulse programing lauguage, PPL, and programs to process, analyze and display the data are written for a Macintosh computer using FORTRAN.

\section{Signa Programs}

Pulse sequence programs were written for the GE Signa ${ }^{T M}$ MR system using the PPL language and compiled with GE's "APPL" compiler. Initial testing used a program called "plotpulses", which simulates the waveforms produced by the gradients, RF amplifier, and data acquisition. Each pulse program has modifiable parameters that direct how the pilses are "played out" and how the MR signal is acquired. Final testing was conducted on the Signa ${ }^{\mathrm{TM}}$ scanner using phantoms (bottles of known boron concentration). This final testing aided in identifying the more sensitive NMR parameters and allowed optimization before the more time critical in-vivo ex. periments.

Specboron.psd. This pulse sequence was modified from a GE Medical System's pulse program, "specfidesip.psd," used for phosphorous, two-dimensional chemical shift imaging (2-D CSI). "Specfidesip" acquires MR data with slice selection, two spatial dimensions (phase-encoded), and one dimension chemical shift. There were two primary modifications to this pulse sequence, "specboron.psd," required by nuclei differences. The long, slice-selective 90-degree pulse of "specfidesip," was replaced with a $100 \mu \mathrm{sec}$, hard, non-selective pulse, and the phase-encode gradient was reduced in time to $500 \mu \mathrm{sec}$. This allowed acquisition of the signal $550 \mu \mathrm{sec}$ after the rf pulse. Other parameters, such as pulse repetition rate, time of echo, and number of averages, can be modified on the SignaTM control screens before program execution. After initial experimentation, many of these modifiable parameters were hard coded into the program.

The phase encoding gradients are incremented in a nested do-loop with one gradient held constant and the other gradient varied through all phase encode levels.
Both $X$ and $Y$ gradients are assigned 16 phase encode levels starting with a negative gradient amplitude. For an axial type scan, the $\mathrm{Z}$ gradient is not used. $\mathrm{Tr} \cdot \mathrm{a}$ ! acquisition time is $16 \times 16 \times$ (\# of averages) $\mathrm{x}$ (repecition time). The number of averages per phase cncode for an optimum signal-to-noise ratio is determined in a spectrosenpy mode. This mods acquires the NMR signal without gradients and, therefore, without signal localization. The repetition time must be greater than the $\mathrm{T} 1$ of the sample nuclei and within the duty cycle requirements of the gradient coil and amplificr. Because this program produced better spectroscopy results than "specrec.psd", it became the program of choice for all spectroscopy experiments.

An axial raw data output file contains 256 free induction decays (FID's) each acquired with a different $X-Y$, phase-encode, gradient combination. This data is processed using the program "2dftcsi" described later. The end result is a three dimensional matrix with two spatial dimensions and one chemical shift dimension which can be displayed in a $16 \times 16$ matrix or by using the peak amplitude of the spectra, the result may be displayed as a color representation of nuclei distribution. The pulse sequence for an axial 2D CSI is shown in Figure 17.

A coronal display can also be obtained by using $Y$ and $Z$ gradients and not using the $X$ gradient. The same thing can be performed using the $X$ and $Z$ gradients and turning off the $Y$ gradient to obtain sagittal displays.

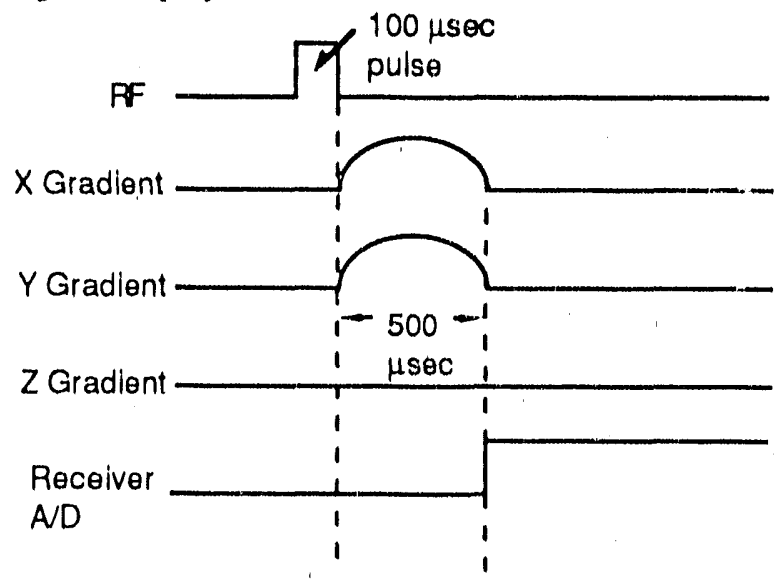

Figure 17. Waveform timing diagram for specboron.psd showing the inputs to the RF power amplifier, gradients and RF receiver. 
Specboron3d.psd. This pulse sequence is very similar to "specboron.psd" except the $\mathrm{Z}$ gradient is now used as an additional phase-encoding gradient achicving spatial encoding in the third dimension or axial direction. The phase-encoding gradients are incremented using a tertiary nested do-loop. Depending on how the gradients are nested, coronal and sagittal images can be obtained. The total acquisition time is $16 \times 16 \times 8 \times$ (\# of averages) $\times$ (repetition time) assuming a spatial matrix of $16 \times 16 \times 8$. The pulse sequence is shown in Figure 18.

Determining the optimum number of averages per phase encode is even more critical with specboron3d.psd as this program will be eight times longer than a 2-D CSI, given the same number of averages per phase encode. Using only 20 averages per phase encode, scan times are about 91 minutes. Scan times for 16 averages per phase encode is about 72 minutes.

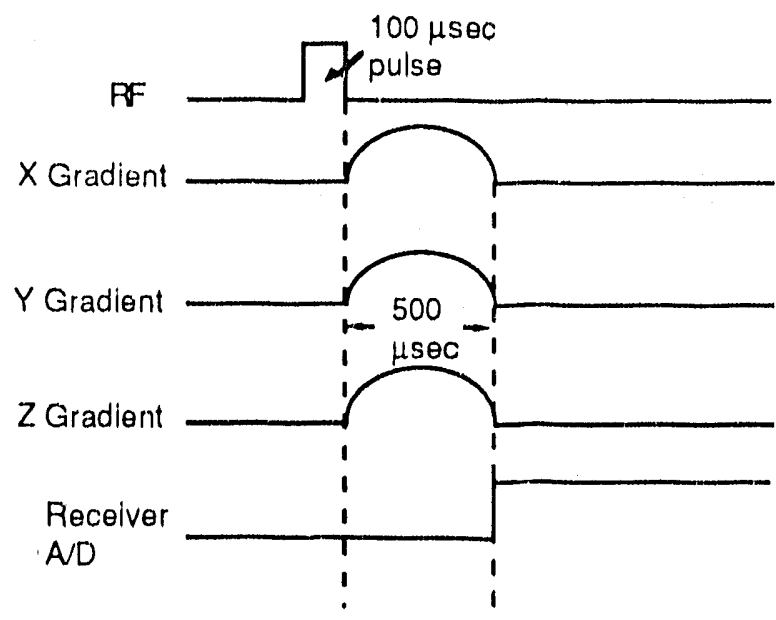

Figure 18. Waveform timing diagram for specboron3d.psd showing the digital inputs of the RF power amplifier, gradients and $R F$ receiver.

Specrec.psd. This program is another pulse sequence for acquiring simple one pulse FID without gradients. After raw data transfer to the Macintosh computer and subsequent processing, only one spectrum is displayed for the entire volume within the RF coil's influence. The repetition rate allowed for pulsing the RF amplifier during this program was tailored for proton spectroscopy and can be increased only to about seven pulses/sec $150 \mathrm{msec}$ repetition rate, TR). It was discovered, however, that using "specboron.psd" in the spectroscopy mode produced better sigral-to-noise ratio (used shorter $\mathrm{RF}$ pulsn widths) and acquired data faster due to a increase in repetition rate of 12.5 pulses/sec ( $8($ msec TR).

Readsigspec. This program transforms spectroscopy files on the Signa ${ }^{T M}$ into a form that can be transferred and read by the Macintosh resident programs.

Kermit. This program is used to transfer files between the Signa ${ }^{T M}$ computer and the Macintosh. Programs are transferred to the Signa ${ }^{T M}$ using the Lext format, and data files are transferred using the binary format.

\section{Macintosh Programs}

Programs for the Macintosh II which process, analyze and display the data acquired with the GE Signa ${ }^{T M}$ system were written in FORTRAN and compiled by software from Language Systems (Virginia). (Step by step instructions to run the Macintosh programs are provided in Appendix C.) The raw data files created by the Signa ${ }^{T M}$ pulse programs can be transferred to the Macintosh using one of three options mentioned under Macintosh connection in the hardware section. Once a raw data file is in the Macintosh environment the following programs may be used:

Readraw. This program converts a Signa ${ }^{\mathrm{TM}}$ raw data file that has been transferred to the Macintosh into a readable form for the " $2 \mathrm{dft}$ ", "3dftcsi", "4dftcsi", and other analysis programs.

3dftesl. This program performs the 3-D Fourier transform on the raw FID data acquired with "specboron.psd" and stores the output file in a format readable by "2dft", "plotcsi", or "tl roi".

4dftesl. This program performs the 4-D Fourier transform on the raw FID data acquired with "specboron3d.psd". This program stores cach of the eight $3-\mathrm{cm}$ thick slices in separate files. Each file can be read by "2dft", "plotesi", or "t1 roi".

Plotesi. This program displays the processed data 
Oi one slice of a 2-D or 3-D CSI experiment, The specira are displayed in scparate boxes according to their spatial registration. An additional option allows this display to be printed.

T1rol. This program superimposos the processed data of one slice of CSI onto a proton image taken in the same plane and field of view as the CSI data.

2dft. This is a general purpose program that performs a Fourier transform on one FID at a time and displays the resultant spectrum.

Mactt. This general purpose program can perform spectral processing, (phase compensation, ctc) on raw or processed data as needed. Detalled analysis can then be performed on a spectrum identifying peak positions, line widths, and areas. 


\section{Operation of the Signa ${ }^{\mathrm{TM}} \mathrm{MRI}$ System}

\section{Setup for Boron Operation}

A primary goal of this task, mentioned at the beginning of this report was to kecp the amount of time needed to change operation from proton to boron and back to proton to under one hour. Presently, a change of operation takes 15 minutes on the average when a routine of module substitution and/or bypassing is used. Although the order in which this stcpup is instituted is not important, making the changes in the same order helps avoid possible mistakes. The following is a step by step procedure for converting the MRI system to boron operation:

1. In the scan (screen) room, unenver the penetration pancl of the screen room (the room where the magnet is located) and remove the head coil transmit cable from $\mathrm{J} 4$ and preamplificr cable from J7. Connect boron preamplificr output to J7 with $50 \Omega$ coax. Attach the boron preamplifier to either preamplifier protection (for linear coil) or quad combine $r /$ splitter with preamplifier protection (for quad coil). Connect head J4 on penctration pancl with an RG-214 coax, or equivalent, to preamplifier protection module or quad combiner/splitter. The coil can be connuted when reeded.

2. In the computer room, disconnect the short cable connecting J108 (RF Amp) from J107 ("atten") on the transceiver patch panel (MR2-A11-J49). Connect the signal input to the Kalmus RF amplifier to the "atten" with either a $20 \mathrm{~dB}$ or 10 $\mathrm{dB}$ in-line attenuator. Connect the "unblank" on the patch panel to the inverter box which has been attacied to the RF gate input of the Kalmus RF amplifier.

3. From the rear of the Signa ${ }^{\top M}$ RF amplifier cabinet disconnect the RF amplifier head coil output (mode 2) from J2 on the RF monitor box (Figure 7) and connect to a $50 \Omega$ dummy load. Connect the output of the Kalmus RF amplifier to $\mathrm{J} 2$ of the RF monitor box with RG-214 coax or equivalent. After a coil has been connected to the quad combiner/splitter or preamplifier protection, switch the inverter (gray box) to on and push the red button on the Kalmus RF amplifier to on.

4. Disconnect the frequency synthesizer control cable (Figure 14) from the rightmost SCM board and install the loop back connector. Set both transmit and receive frequency synthesizers to the boron frequency.

5. Position RF coil as needed using the alignment lights. Connect a linear coll to the preamplifier protection, or a quad coll to the quad hybrid combincr/splitter. If proper operation is not achieved, or the signal-to-noise is too low, check the resonant frequency of the loaded coil with the HP. 4193 A vector impedance analyzer.

Conversion back to proton requires only a reversal of Stepr 1-5. To ensure the system will continue in work clinically, a proton scan should be taken with the quad coil phantom and quad head coil. A normal head imaging profile can be used.

\section{Operation}

Operation of the Signa ${ }^{\top M}$ MRI system is controlled by the touch pancls on the system cansole. The system must be in the rescarch mode as opposed to the clinical mode. A special password is required to enter the research mode after initializing a new study. (Some systems require only a change in imaging mode.) Special licensing is given by GE Medical Systems to access the research mode and to obtain the password. After entering the research mode the fol: lowing are representations of screens presented at the system console to the operator with the button to be touched on each screen darkened: 


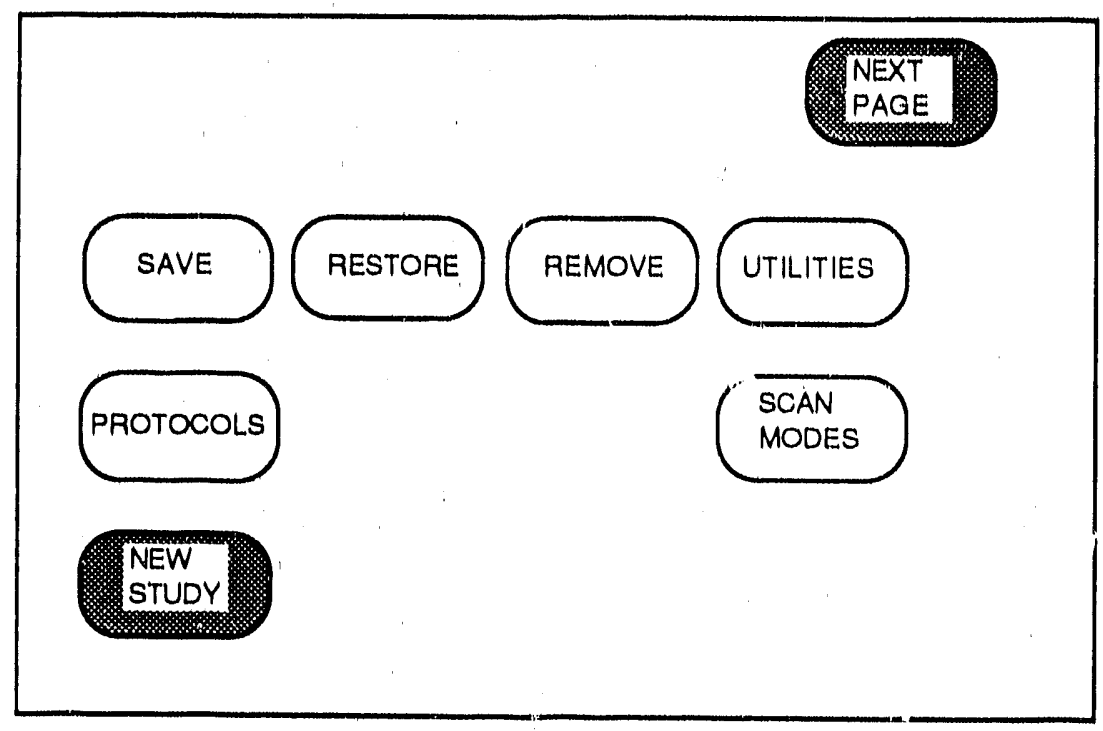

Touch "next page" button in upper right hand corner to ad vance to next screen. (All screens show next page and/or previous page)

\section{Screen Two (2)}

Answer the following:

Id (passwerd))(If the research mode has already been entered, the "Id" can be of your choosing) Weight 110 Monitor SAR? N

Screen Three (3)

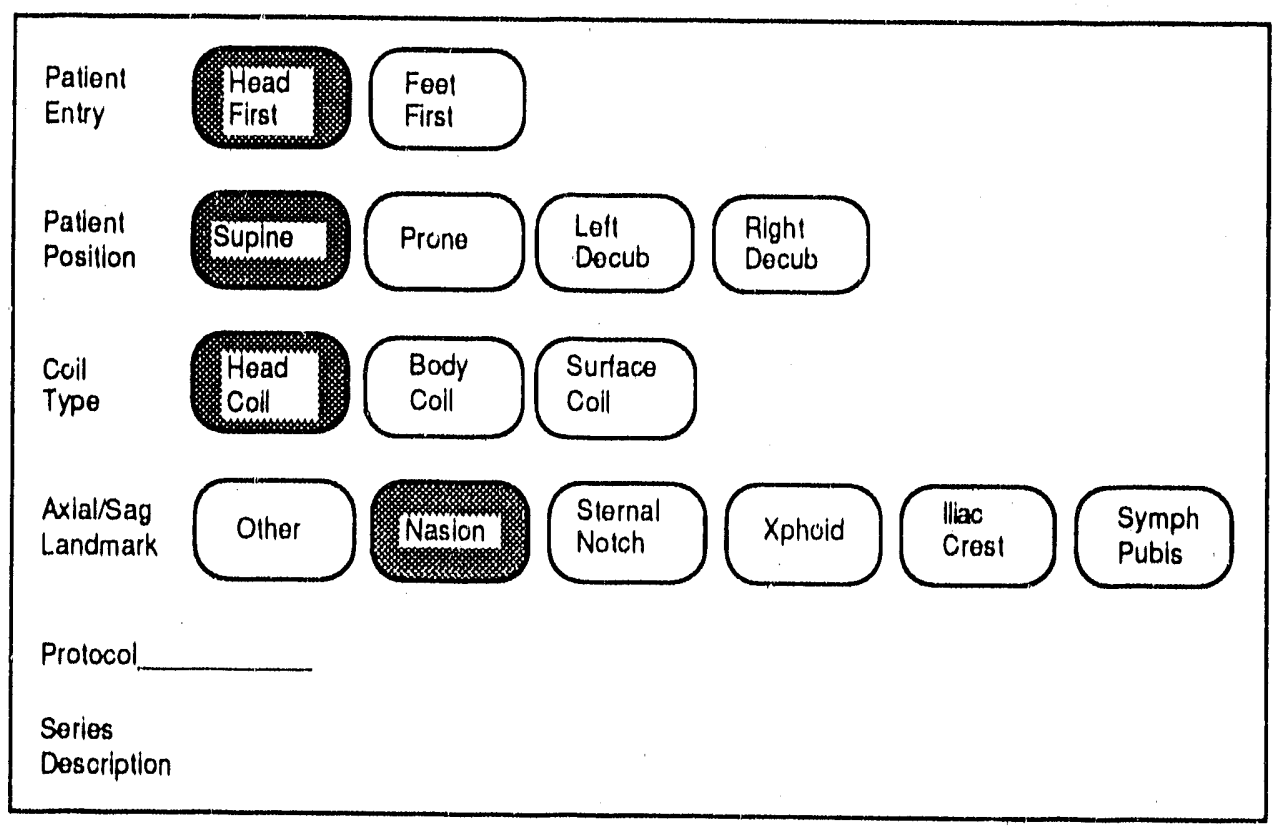




\section{Screen Four (4)}

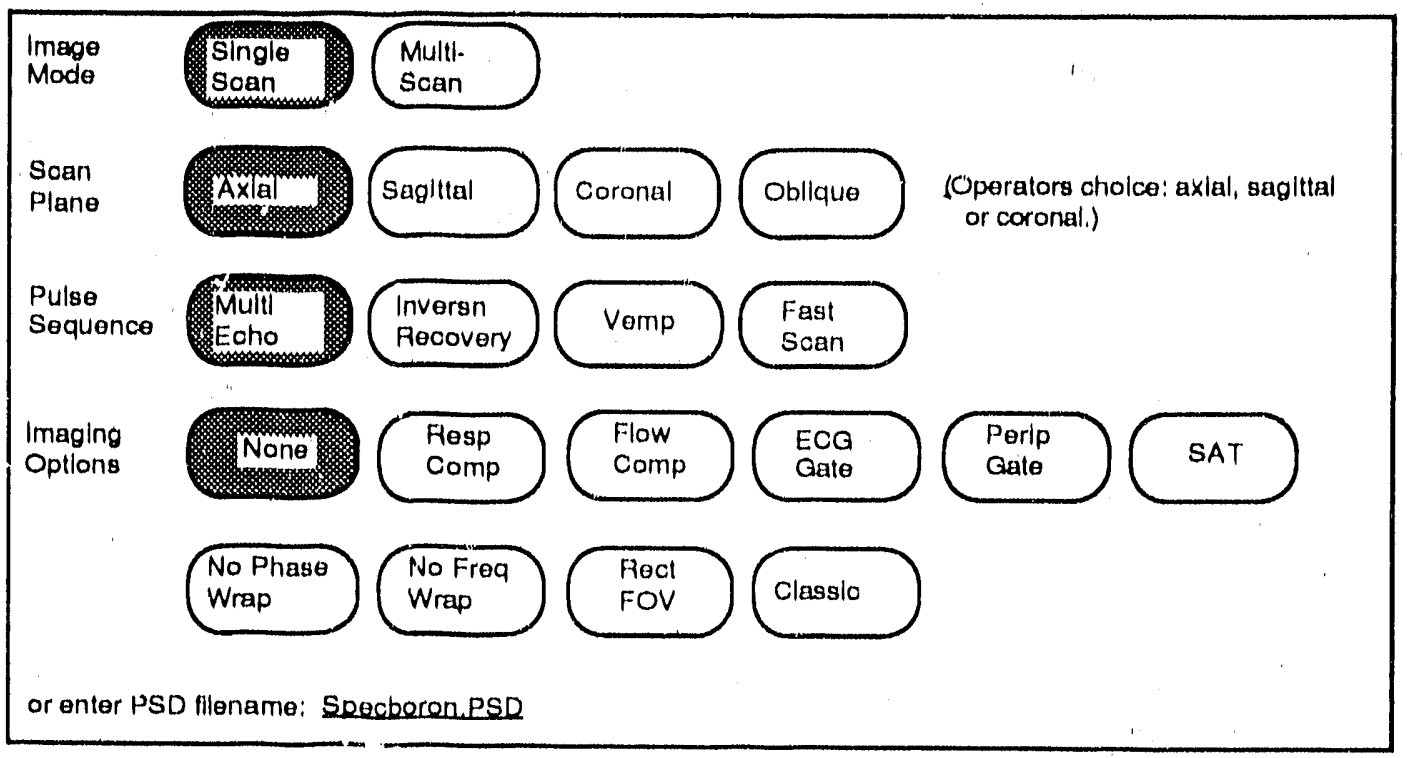

\section{Screen Five (5)}

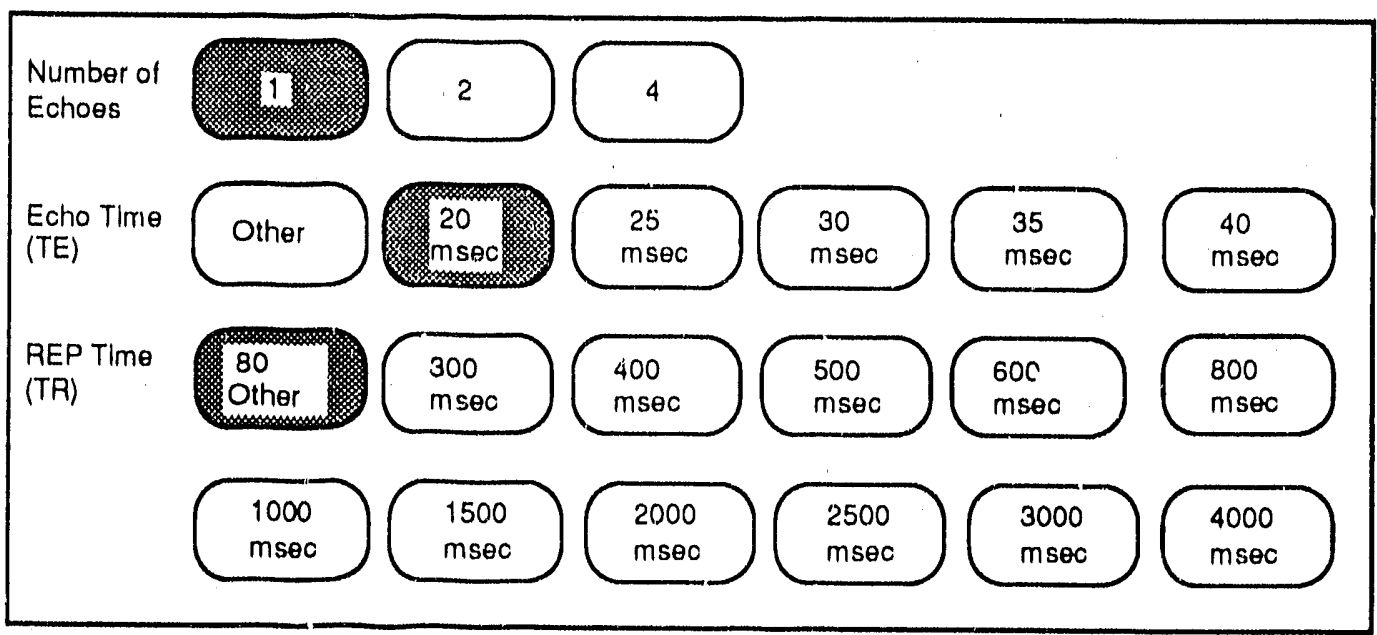




\section{Sicreen Six (6)}

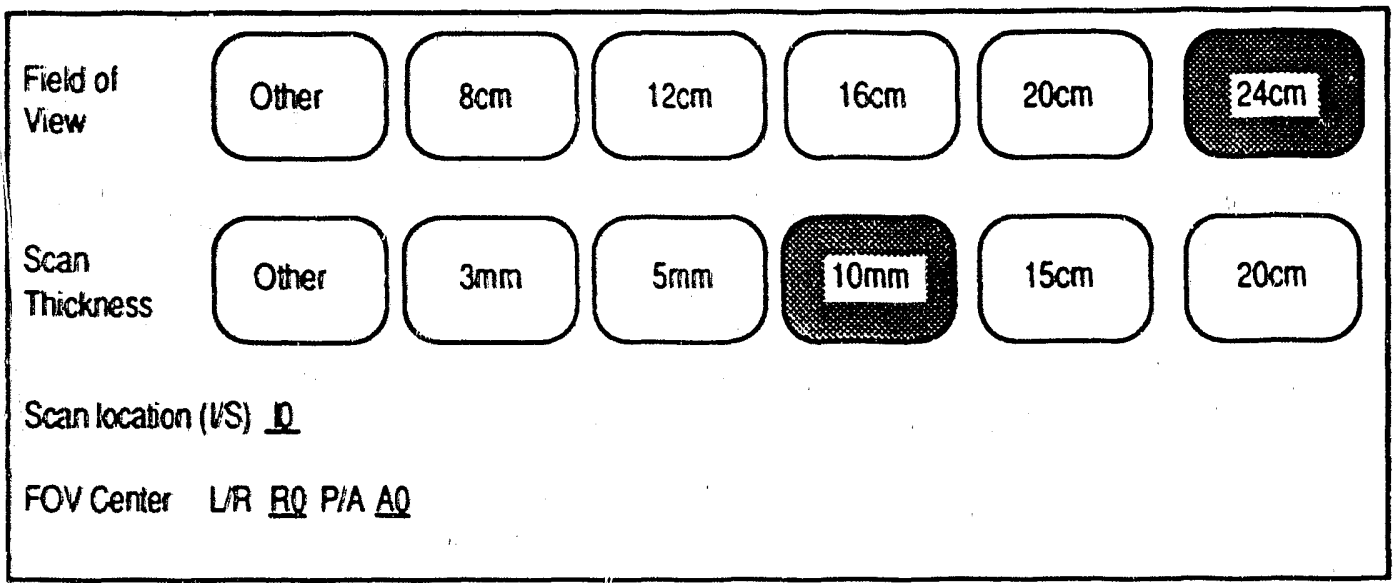

\section{Screen Seven (7)}

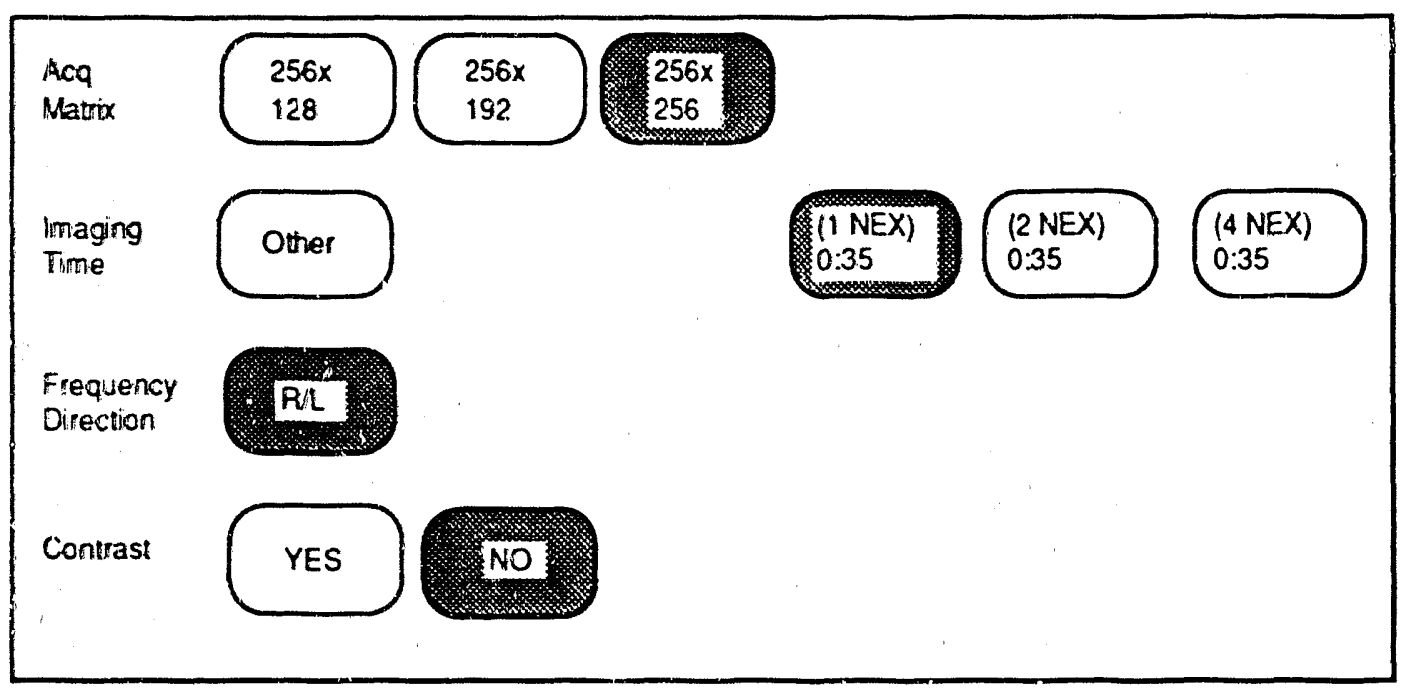




\section{Screen Eight (8)}

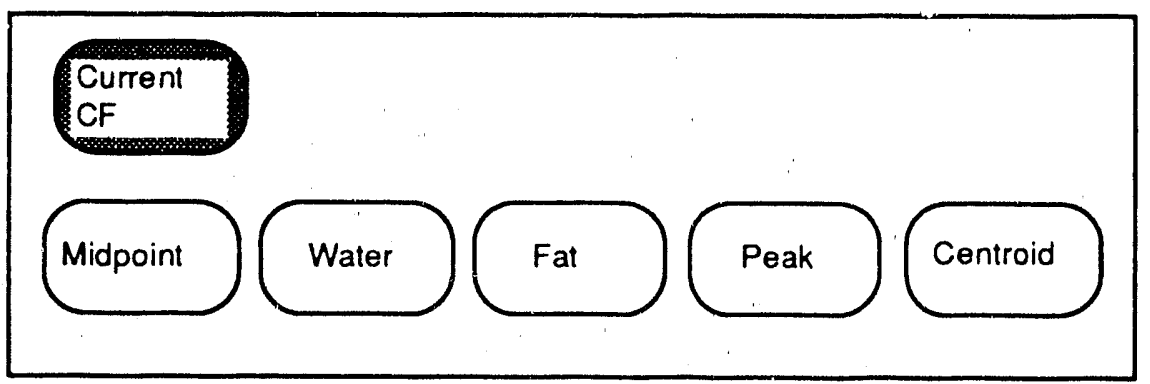

\section{Screen Nine (9)}

This screen list all the selections that have been chosen so far. If a mistake has been made, touching the small square next to the wrong parameter will bring up the screen where the selection was made and the correct parameter can be entered.

\section{Screen Ten (10)}

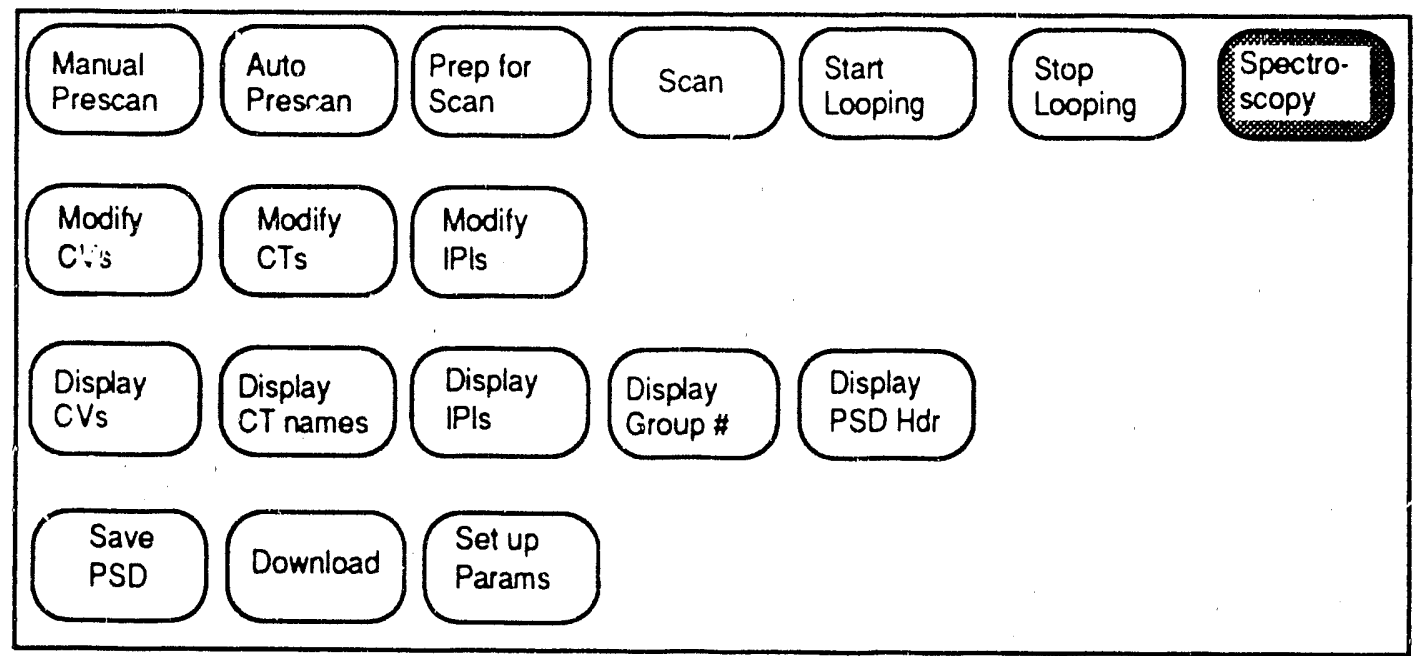




\section{Screen Eleven (11)}

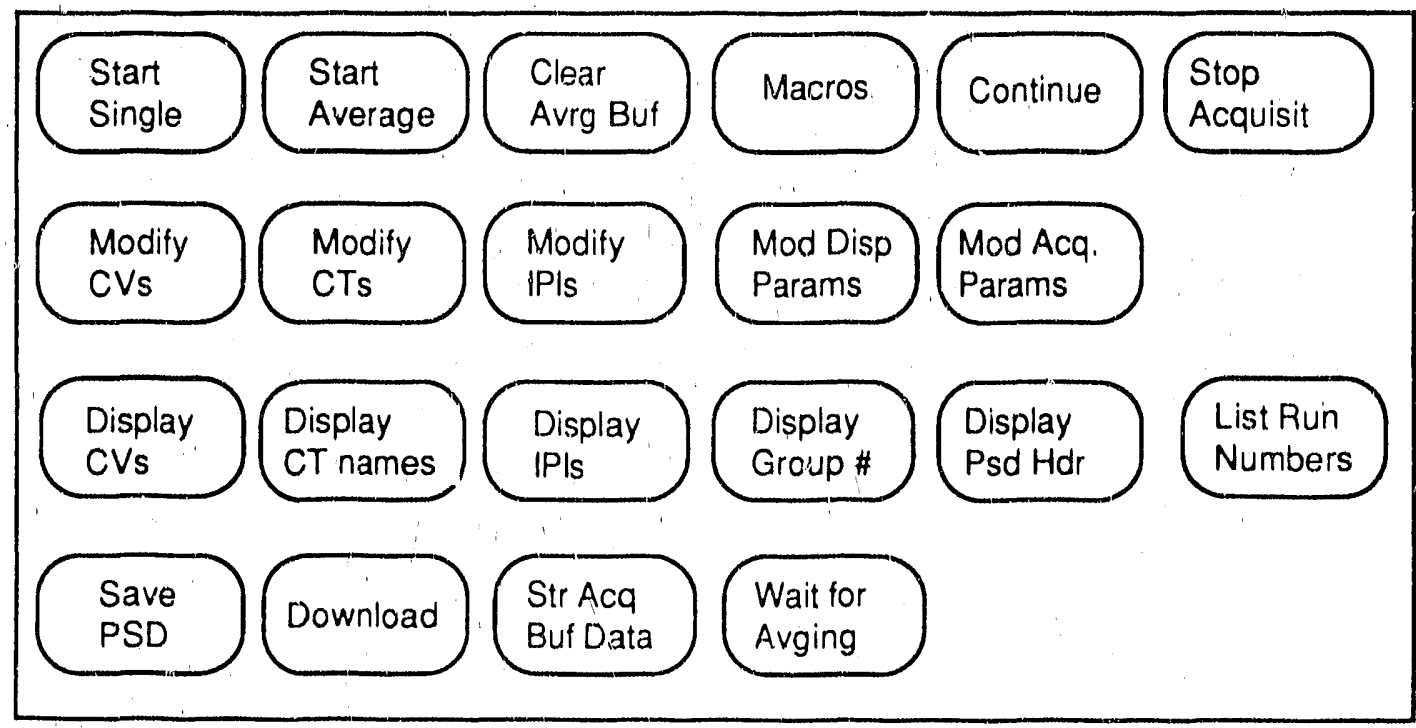

After the spectroscopy mode is entered, screen 11 will appear where most of the modifications to the pulse program will be en:ered. Control variables must be changed by first touching the "Modify CVs" button, or typing MCV followed with a return, and then typing the control variable to be changed followed with a return. The following control variables are changed as follows:

$\begin{array}{ll}\text { op_tr } & .8 \mathrm{E} 6 \text { (usually entered from touch } \\ \text { screen) } \\ \text { spec_width } & \begin{array}{l}10000 \text { (can be } 5000 \text { after signal has } \\ \text { been optimized) }\end{array} \\ \text { nav } & \begin{array}{l}64 \text { (depends on how many samples } \\ \text { required) }\end{array} \\ \text { yres } & 2048 \text { (if doing boron3dcsi.psd) }\end{array}$

(To exit the MCV mode, touch the "Modify CVs" button or just retum from the key board.)

A nav (number of averages) of 64 for "specboron" (2-D CSI) results in a scan time of 36 minutes and 25 seconds. A nav of 16 for "boron3dcsi.psd" results in a scan time of 72 minutes and 45 seconds. Doubling the number of averages doubles the scan time. White in the spectroscopy mode, nav is not applicable, and "Start Single" will show only the results of one pulse. A running average is maintained with "Start Average" and continues until terminated by a "Stop Acquisit." The number of averages will be displayed at the bottom of the screen. To store this data, touch "Str Acq Buf Data." A description and name will be requested for this data. Once this information is entered, the data file will be given a $N^{* * *} .001$ file name for later retrieval.

Before any acquisition can begin, the transmit and receive attenuation must be set. "This is accomplished by touching the "Mod Acq Params" button. Another screen will appear listing RA (receive attenuation), TA (transmit attentuation), frequency (not applicable for boron), and RF (RF bandwidth). The following are nominal values for spectroscopy and specboron:

RA 0

TA 60-80 (this depends on the coil used and an in-line attenuation of $10 \mathrm{~dB}$ for quad coil. TA can be reduced by $500.1-\mathrm{dB}$ steps or less.)

RF $5 \mathrm{kHz} \quad$ (cxit pagc)

TA determines the 90-degree RF pulse and must be obtained by trial. The results of changing TA on the spectrum will be seen on the middle display of the control console. The three windows of this middle display (Figure 19) can be changed by touching the "Mod Disp Params" button and entering the following values from the keyboard:

MW 1

$\mathrm{CP}$ 
MW 2

CP

WA 9

$\mathrm{VZ}$

VO - 50

VM 5 (this number depends on the strength of the signal)

MW 3

CP

$\mathrm{VZ}$

VM 5 (this number depends on the strength of the signal)

Explanation of these codes is displayed when in the "Mod Disp Params" mode. In general, MW=modify window, $\mathrm{CP}=$ connect points, $\mathrm{WA}=$ waveform to be displayed, $\mathrm{VZ}=$ =vertical zoom, $\mathrm{VO}=$ vertical offset, and $V M=v e r t i c a l$ multiplier (changes the display gain). Exiting "Mod Disp Params" mode will save all of these settings.

Signal averaging can now be started by touching "Start Average." If everything is set up properly, a spectrum will appear in windows 2 and 3 , and the FID will appear in window 1 . Changing the TA setting will modify the height of the spectrum seen in windows 2 and 3. This height must be maximized for the best signal-to-noise. A horizontal line can be selected from the console and put on the screen for comparison purposes. This line, appearing in window 2 , is only an aide for maximizing the signal and does not modify the signal. The averaging buffer is cleared and the peak disappears every time TA (or $\mathrm{RA}$ ) is changed.

Once all parameters have been optimized, a spectroscopy session can be initiated with "Start Average." A 2-D CSI or 3-D CSI scan (depending on which PSD is being executed) can be started by exiting the spec'roscopy screen (Exit page) and by touching "Scan." 


\section{Imaging and Spectroscopy Results}

Since this technology is being used as an investigative tool on a continuing basis, only a few examples of the imaging and spectroscopy results will be reported demonstrating its capability.

\section{Spectroscopy}

Window 1 (I-Channel Raw seen in Figure 19) shows the FID signal which has just been acquired and averaged with previous acquisitions. Window 2 (Magnitude Spectrum) shows the magnitude of either the I or $\mathrm{Q}$ channel (determined by user) without phase information. Window 3 (Pure Absorption) st.uws the absorption or the actual signal with phase information. Window 4 (Peak $A / D$ ) gives the user information on the analog-to-digital $(A / D)$ converter's usage. The $A / D$ converts the FID signal to digital information readable by the computer. A reading of $100 \%$ means the full range of the $\mathrm{A} / \mathrm{D}$ is being used. To prevent the $A / D$ from overflowing this range use the RA command from the "Mod Acq Params" screen and adjust $A / D$ usage to about $30-60 \%$. The larger $\mathrm{RA}$ is, the smaller the $A / D$ range usage will be. Setting the A/D range to less than $60 \%$ allows for large signals and prevents overflows.

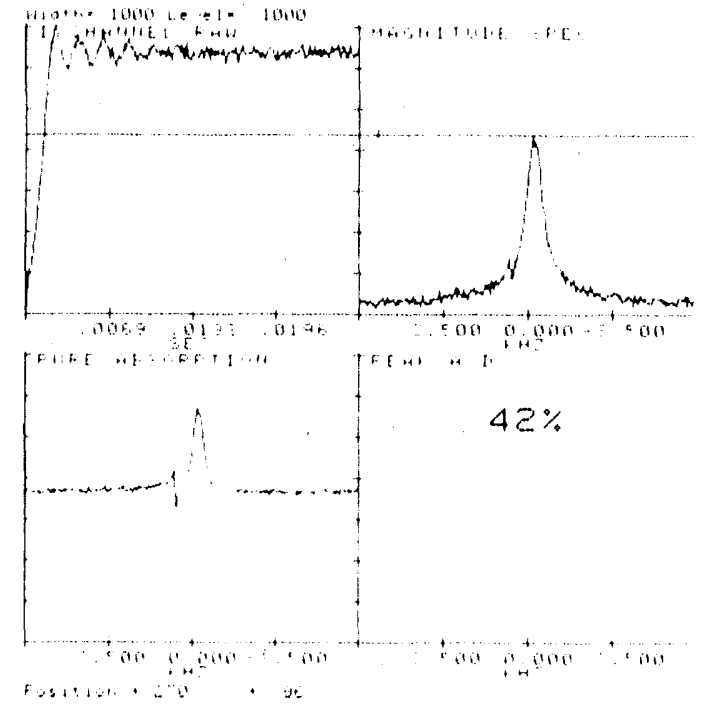

Figure 19. Spectroscopic results displayed on the control console.
A series of spectra from the head of a $53 \mathrm{lb}$ Collic was acquired on the Signa ${ }^{\mathrm{TM}}$ and analyzed on the Macintosh by the "macft program." After the phase was corrected, the area under a spectrum curve can be obtained. This area is proportional to the boron concentration. Serum data was also taken in between spectra arquisitions and analyzed by inductively coupled plasma atomio emissions spectroscopy (ICPAES). These data are displayed in Graph 1. The information obtained with this study forms the basis for all proposed pharmacokinctic studies.

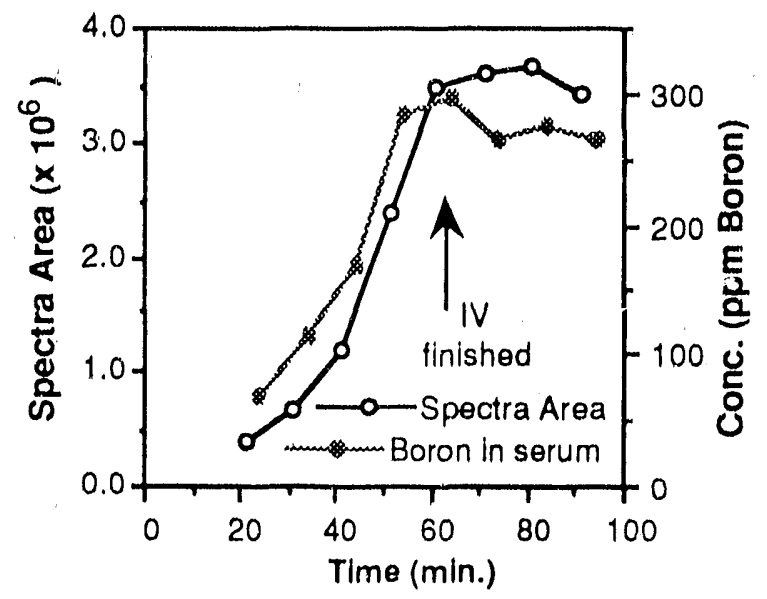

Graph 1. Spectroscopic data showing the boron uptake in muscle tissuc within the brain and concentration of boron in scrum of a $53 \mathrm{lo}$ Collic.

With a known boric acid concentration reference within the ficld of view (FOV), the spectrum area of the BSH peak can be correlated to conceritration for either the whole head spectroscopy or CSI scans. This technique was demonstrated in 1987 by Dr. Todd Richards and reported to the Radiological Society of North America (RSNA). ${ }^{9}$

Using "specboron.psd," a 2-D CSI was taken of a 25 pound nontumor Beagle dog. and is shown in Figure 20. This data represents 64 averages per phase encode. Scannirig time was 36 minutes and 25 seconds. 


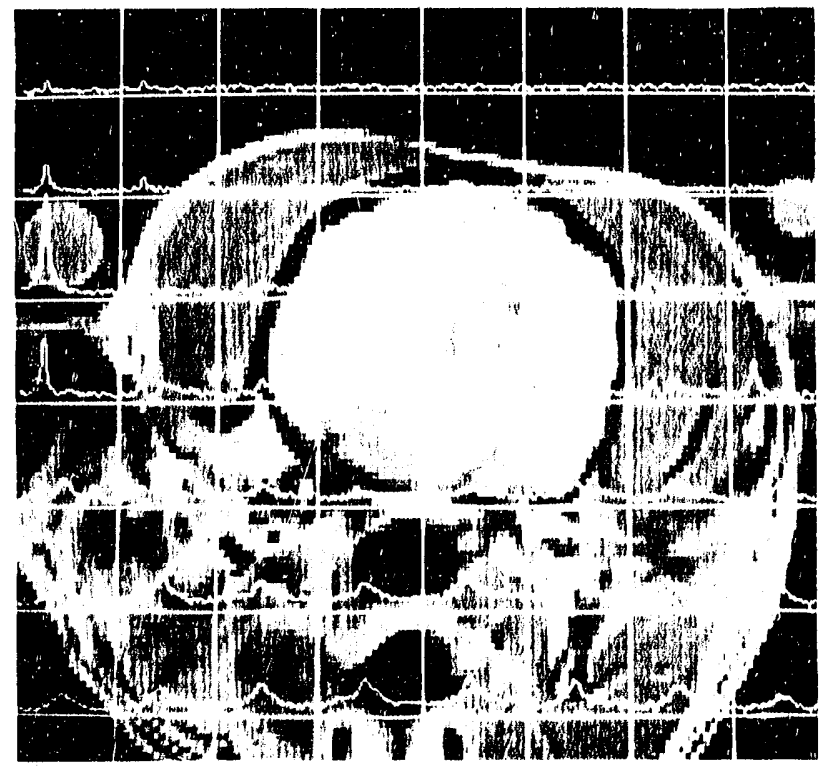

Figure 20. 2-D CSI axial image superimposed over a proton image of the head of a $25 \mathrm{lt}$ Beagle.

\section{2-D CSI}

The results of this 2-D CSI with the 2-D CSI data superimposed over a color proton image of a dog head (Figure 20) is an axial scan with all signal data integrated along the axial (Z) direction, leaving only $X$ and $Y$ data for locating horon. Without $Z$ direction information, it is difficult to determine where the boron signal originates. A boron signal emanating from within the muscle tissue which surrounds the dog's brain will appear to be coming from the brain. For this reason the 3-D CSI images give a better perspective of boron within the FOV. The 3-D CSI image, shown as ar overlay to a proton image in Figure 21, gives more location information.

\section{3-D CSI}

Both the 3-D CSI and proton image of Figure 21 were taken of a 60) lb Basset Hound with a small pituitary tumor located at the base of the brain. This figure stows a lack of the boron signal in the brain, but some signal coming irom the pituitary tumor. The 3-D CSI information is displayed as once slice from a series of eight axial slices, calch with a thick. ness of $3 \mathrm{cmi}$. Each "tox" (voxel) of signal represents about $1.5 \mathrm{~cm} \times 1.5 \mathrm{~cm} \times 3.0 \mathrm{~cm}$ of the head. Because of gradient amplifier current limitations, the FOV of all imaging techniques must be at least 24 cm or greater. A smaller FOV requires more gradient magnetic field and consequently more current flowing through the gradient coil.

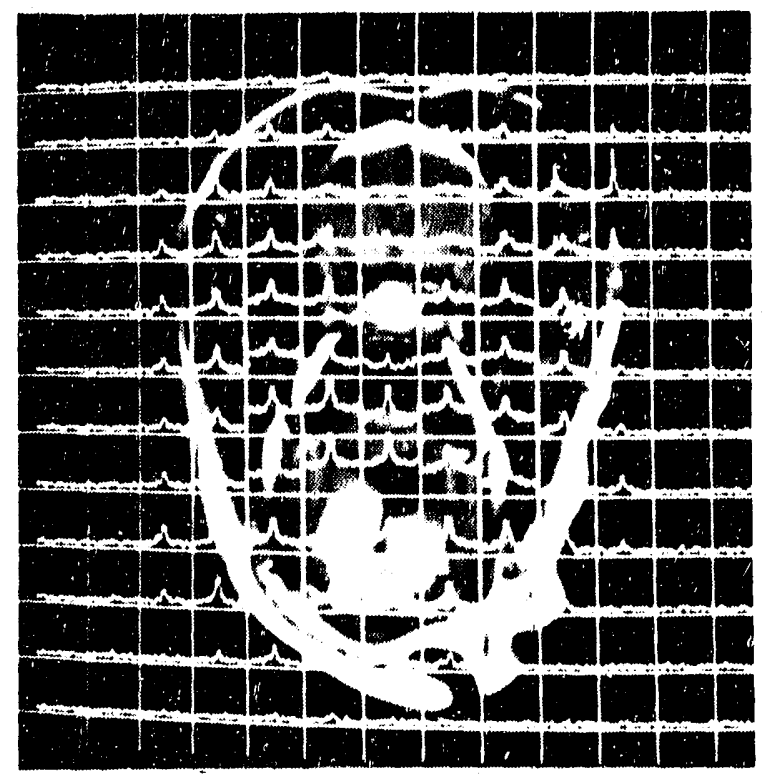

Figure 21. 3-D CSI axial image superimposed over a proton image of the flead of a dog with. pituitary lumor. 


\section{References}

1. I. L. Pykett, "NMR Imaging in Mcdlcine," Sci. Amer. , 1982, May, pp. 78-88.

2. Impedance Matching Networks Applied to RF power transistors, AN-721, Motorola, Inc., 1974, p. 4-5.

3. C. E. Hayes, W. A. Edelstein, and J. F. Schenck, NMR in Medicine, (S. R. Thomas and R. L. Dixon, Eds.), Amer. Assoc. Physicists Med., New York, 1986, pp.142-165.

4. D. I. Hoult and P. C. Lauterbur, J. Magn. Reson., 34, 1979, p. 425.

5. D. I. Hoult, Prog, NMR Spectro., 12, 1978, p. 41.

6. C. L. Alley, K. W., Atwood, Electronic Engineering, third edition, John Wilcy and Sons, New York, pp. 313-314.

7. C. N. Chen, D. I. Houll, and V. J. Sank, J. of Magn Reson. 54, 1983, p. 324.

8. C. E. Hayes, W. A. Edelstein, J. F. Schenck, O. M. Muller, and M. Eash, "An Efficient, Highly Homogeneous Radiofrequency Coil for Whole-Body NMR Imaging at $1.5 \mathrm{~T}, " \mathrm{~J}$. of Magn. Reson., 63, 1985, pp. 622-628.

9. T. L. Richards, P. R. Gavin, S. L. Kraft, M. L. Gricbenow, and R. N. Golden, "11B NMR spectroscopy of excised mouse tissue after infusion of boron compound used in neutron capture therapy" (Abstract), Radiology, 165, 1987, p. 346. 


\section{APPENDIX A \\ BROADBAND RF POWER AMPLIFIER SPECIFICATION}




\section{Specification for a Broadband RF Power Amplifier}

Peak Output Power

Frequency Range

Maximum Pulse Width

Duty Cycle

Linearity

Droop

Power Gain

Gain Variation

Rise Time

Fall Time

Maximum Input VSWR

Unblank Time

Blank Time

Noise Figure

Harmonics

power.

AC Power Requirements

$\mathrm{RF}$ Input Conricctor

RF Output Connector

Cooling

Diagnostics

Operating Environment

Non-Operating Environment

Mounting
2.0 KW at $1 \mathrm{~dB}$ compression point

$6.30 \mathrm{MHz}$

$13 \mathrm{msec}$

$20 \%$ at rated peak power and maximum pulse width. Within $\pm 1.0 \mathrm{~dB}$ of idcal dBm-dBm characteristic from rated power to $40 \mathrm{~dB}$ below rated power.

$<0.25 \mathrm{~dB}$ during $6.0 \mathrm{msec} 20 . \mathrm{KW}$ reclangular pulsc. Output power of $2.0 \mathrm{KW}$ shall be provided for input drive not greater than $0 \mathrm{dBm}$ and not less than $-8^{\prime}$ $\mathrm{dBm}$.

$\pm 0.15 \mathrm{~dB}$ over any $250 \mathrm{KHz}$ band.

$<1 \mu \mathrm{sec}$

$<1 \mu \mathrm{sec}$

$<1.5: 1(50 \Omega)$

$<2(0) \mu \mathrm{sec}$

$<10 \mu \mathrm{sec}$

$<12 \mathrm{~dB}$

$<-30 \mathrm{~dB}$ from rated power to $45 \mathrm{~dB}$ below rated

$208 \mathrm{~V}, 50 / 60 \mathrm{~Hz}$

BNC (Female)

Type N (Femalc)

Forced air.

Overtemperture, overcurrent.

Ambient temperature $=18$ to 30 degrees $\mathrm{C}$

Humidity $=30-60 \%$ non-condensing

Magnetic Ficld $=50$ gauss

Temperature $=-34$ to 60 degrees $\mathrm{C}$

Humidity $=95 \%$ non-condensing

Self-cnclosed 
APPENDIX B

RF POWER AMPLIFIER MANUAL

B. $\sqrt{2}$ 


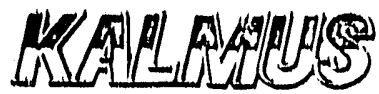

RF POWER AWPINERS

TECHNICAL MANUAL

MODEL 169LPL

HI-POWER PULSED AMPLIFIER 
FINAL TEST DATA

Model 169LPL

Serial No. 09208-1

Description RF POWER AMPLIFIER

Date $9-20-88$

Invoice No. 92695

Customer

EG\&G IDAHO, INC.

Frequency Range $\_-30 \mathrm{HHz}$ Max Pulse Width

Power Gain $\quad 6.2 d 6$ men Max Pulse Duty

Gain Flatness $\pm 2 d B$ Blanking Speed

Power Output $4 K^{\prime}$ Max Dynamic Range

* Harmonics Zed B $(13 \cdot 30 \mathrm{mHz})$ VSWR Set

VSWR Set $N^{\prime} / A$ Primary Power Zed ac

* 6.17 mitt external jitter regiecued

INSPECTION CERTIFICATION:

KALMUS ENGINEERING INTERNATIONAL certifies that all applicable standards of materials and. workmanship have been fully complied with during the manufacture of this device and that the finished product meets or exceeds the company's final test and quality control specifications standards.

Witnessed by:

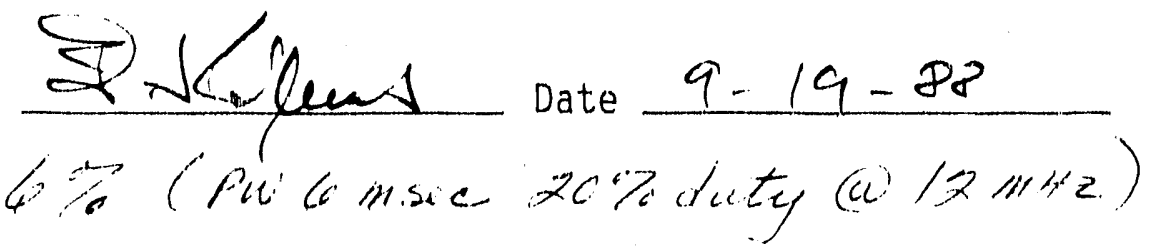

B-4 
CERTIFICATE OF COMPLIANCE

CUSTOMER___EG\&G IDAHO, INC.

P.O. NO:

\#C88312458

W.O. NO:

$\# 2475$

It is hereby certified that the equipment shown below conforms to the requirements of your purchase order and is constructed from all new materials and in accordance with accepted industry workmanship practices. The measured performance meets or exceeds the applicable specifications except as follows:

Equipment delivered under this document:
DESCRIPTION
PART NO.
SERIAL NO.
RF POWER AMPLIFIER
MODEL 169LPL
09208-1

PART NO: MODEL 169LPL 


\section{TABLE OF CONTENTS}

ITEM

PAGE NUMBER

GENERAL INFORMATION

Equipment Description

1

Specifications

PLACING EQUIPMENT IN OPERATION

CIRCUIT DESIGN

Peak Detector

Signal Blanker

Blanker Regulator

Reference Regulator

Meter Circuit

Pulse Width Limiter

25 Watt Preamp/Driver

Power Output Amplifier

Power Supply

MAINTENANCE

Cooling

6

Fuses

. A L 


\section{GENERAL INFORMATION}

EQUIPMENT DESCRIPTION

The Model 169LPL is a wideband pulse amplifier, covering an instantaneous frequency range from $6-30 \mathrm{MHz}$; at a power output level up to 4000 Watts into a $50 \mathrm{Ohm}$ load: MOS-FETS are used throughout the entire circuitry. They provide good performance and reliability for a modest increase in cost:

Having an overall system gain of $63 \mathrm{~dB}$, the 169LPL is ideally suited for wideband application. The amplifier is compatible with most RF signal generators capable of $3 \mathrm{~mW}$ of signal output. Although the system has no special provisions designed in for VSWR protection, the MOS-FETS by themselves exhibit high tolerance to load mismatches: It is suggested; however, to keep the load VSWR below 3:1 at full power output.

A meter has been added to the front panel to facilitate monitoring the amplifier output signal, as well as voltage and current checks: The upper scale is used to measure peak power (Watts) into 50 ohms and the lower scale provides a convenient readout for DC Volts and Amperes used as a self check feature: ( $D C$ current readings are not absolute, and are for reference only): 
SPECIFICATIONS MODEL \%69LPL

HI-POWER PULSE AMPLIFIER

Rated Linear Power

Frequency

Gain

Maximum Pulse Width

Signal Rise and Fall Time

External Gating - Rise Time

- Fall Time

Duty Cycle

Pulse Droop (at $2 \mathrm{KW}$ )

Noise Figure

Input Impedance

Output Impedance

Input VSWR

Output VSWR

Power Requirements

$3 r d$ order Intermodulation

Intercept Point

Harmonics (at $2 \mathrm{KW}$ )

Size $(H \times W \times D)$

Weight

RF Input Connector

RF Output Connector

External Gating

External Gating Connector

Rack Mounting

Metering

Cooling (Internal)
: $4 \mathrm{KW}$ Peak Pulse Maximum

: $6-30 \mathrm{MHz}$

: $63 \mathrm{~dB}$ Typical

: 15 Milliseconds (Auto-Limit)

: 1 Microsecond Maximum

: 1.5 Microseconds (Blanking)

: 1.5 Microseconds (Blanking)

: 20\% Maximum (Full Output)

: 5\% Typical

: $8 \mathrm{~dB}$ (Typical)

: 50 Ohms

: 50 Ohms

: Less than 1.5:1

: Less than 2.0:1

: Power Supply Taps Provided for 208 VAC $\pm 10 \%$ 50-60 Hz, Single Phase at 10KVA Peak Power Maximum

: $\quad+73 \mathrm{dBm}$ (Typical)

: $20 \mathrm{~dB}(13-30 \mathrm{MHz})$ $6-13 \mathrm{MHz}$ (External Filter Req:)

: $28 \times 22 \times 28$ Inches

: 260 Lbs. (Incl. Cabinet)

: BNC (Front Panel)

: Type "N" (Front Panel)

: TTL Compatible (Positive True)

: BNC (Front Panel)

: Provided

: All Vital Circuits Including Peak Power

: Self-Contained Forced Air 
PLACING EQUIPMENT IN OPERATION

CAUTION: Be sure that the gating input signal is TTL compatible, active low. (Less than 0.7 Volts gates the amplifier "OFF". Greater than 2.2 Volts gates the amplifier "ON") Also be sure that the duty cycle of the gating input signal (the percentage of time that the gate signal is high) does not exceed $20 \%$.

Connect a 50 Ohm load capable of handling 500 Watts of $\mathrm{CW}$ power to the output conrector. Connect a $50 \mathrm{Ohm}$ signal source (Minimum of $3 \mathrm{~mW}$ ), to the signal input connector. Reduce the signal source output to minimum: Connect a TTL compatible, active high gating signal of less than $20 \%$ duty cycle to the gate input connector. Verify that your AC line voltage agrees with the internal wiring of the 169LPL. (Note the serial number plate.) Turn equipment "ON". The AC "ON" light should illuminate and the fan (blower) should be heard clearly picking up speed:

Watch the panel meter (set to peak power) and increase the signal source output until a meter reading is observed: If your load is a good $50 \mathrm{Ohm}$ load, you can read the output power directly off of the meter scale.

CAUTION: NEVER RUN FULL POWER WITH THE LOAD DISCONNECTED. THIS MAY CAUSE PERMANENT DAMAGE TO THE OUTPUT TRANSISTORS.

\section{CIRCUIT DESIGN}

The system consists of nine basic blocks:

1. Peak Detector

2. Signal Blanker

3. Blanker Regulator

4. Reference Regulator

5. Meter Circuit

6. Pulse Width Limiter

7. 25 Watt Preamp/Driver

8. Power Output Amplifier

Q: Power Supply 
PEAK DETECTOR (A'7)

This circuit is located on a plug-in circuit board in the $P=A$ : enclosure. It receives a small RF detected $D C$ pulse voltage (50 - 200MV) and processes it into a slow-acting DC current capable of holding the $D C$ meter movement steady at the peak of the pulse level, thus permitting accurate peak pulse power measurements from $100 \mathrm{milliseconds}$ down to $5 \mathrm{microseconds}$, and up to $50 \%$ duty cycle.

SIGNAL BLANKER (A10-13)

This circuit is located on a plug-in printed circuit board in the P.A. enclosure. Its primary function is to establish a low-impedance pulse source for driving the gates of the FET'S from class "A" to "OFF" condition, in a very fast time (Less than 2 microseconds): Four outputs are provided to feed four output devices. Maximum output swing of the pulse is 5 Volts.

BLANKER REGULATOR (A8-9)

This is an adjustable voltage regulator which controls the gate drive to the amplifier during blanking pulses. This assembly is located on the baseplate of the power supply enclosure.

REFERENCE REGULATOR (A15)

This circuit is located on a plug-in printed circuit board in the power supply enclosure: It uses a reference diode, buffered by an emitter follower transistor, to provide a low current reference voltage for the power supply.

METER CIRCUIT

A front panel meter enables the operator to monitor two $D C$ supply voltages, eight DC currents and also peak RF power in watts. The upper scale is calibrated from 0 - 6000 Watts peak into 50 ohms. The lower scale is $0-75$ Volts or $0-75$ relative current. Accuracy should be $10 \%$. 
PEAK DETECTOR (A7)

This circuit is located on a plug-in printed circuit board in the P.A. enclosure. It receives a small RF detected $D C$ pulse voltage (50-200MV) and processes it into a slow acting DC current capable of holding the $O C$ meter movement steady at the peak of the pulse level, thus permitting accurate peak pulse power measurements from 100 milliseconds down to $5 \mathrm{microseconds}$, and up to $50 \%$ duty cycle.

SIGNAL BLANKER (A 10-13)

This circuit is located on a plug-in printed circuit board in the P.A. enclosure. Its primary function is to establish a low-impedance pulse source for driving the gates of the FET'S from class "A" to "OFF" condition, in a very fast time (Less than $\mathbf{2}$ microseconds). Four outputs are provided to feed four output devices. Maximum output swing of the pulse is 5 volts.

BLANKER REGULATOR (A 8-9)

This is an adjustable voltage regulator which controls the gate drive to the amplifier during blanking pulses. This assembly is located on the baseplate of the power supply enclosure.

REFERENCE REGULATOR (A 15)

This circuit is locited on a plug-in printed circuit board in the power supply enclosure. It uses $2 \in E E R E \tilde{Z} \mathcal{E}$ diode, buffered by an emitter follower transistor, to provide a low current reference voltage for the power supply.

METER CIRCUIT

A front panel meter enables the operator to monitor two DC supply voltages, eight $D C$ currents and also peak RF power in watts. The upper scale is calibrated from 0 - 6000 Watts peak int 50 ohms. The lower scale is 0 - 75 volts or 0 - 75 relative current. Accuracy should be $10 \%$. 
PULSE WIDTH LIMITER (A6)

The Pulse Width Limiter circuit receives an externally supplied positive going TTL level blanking pulse, and limits this pulsewidth to $15 \mathrm{milliseconds}$ duration. This assembly is a printed circuit board which is mounted on the baseplate of the $P$ : $A$. enclosure. The incoming pulse triggers a monostable multivibrator whose duration is set by an R-C time constant. The blanking pulse is "Anded" with and therefore limited by, the output of the multivibrator.

The Pulse Width Limiter circuit also provides an output to drive an LED which indicates excessive pulse width input:

\section{WATT PREAMP/DRIVER (A1)}

The preamp has approximately $40-45 \mathrm{~dB}$ of system gain. Its frequency bandwidth is in excess of $100 \mathrm{MHz}$. Power output is 25 Watts minimum: It is lcoated on the right hand wall of the system: It runs class "A".

\section{POWER OUTPUT AMPLIFIER (A2-5)}

The power output amplifier consists of sixteen identical power transistors (MOS-FET), tied together at their input by power splitters, and in reverse again at their outputs by power combiners: The gain is approximately $22 \mathrm{~dB}$ average, depending on the frequency. This final gain requires $10-25$ Watts of drive power to produce 4000 watts maximum of output power. The power splitter/combiner assembly is mounted on a heatsink located on the heat dissipator assembly: The output combiner couples directly to an RF sampler/ detector which provides a signal for use by the peak detector board.

POWER SUPPLY

The power supply consists of four voltage regulators: BR1 thru BRA fullwave rectifier bridges, with $C 1$ - $C 4$ capacitors provide the $D C$ power for the $+42 \mathrm{~V}$ regulators: $Q 1-Q 4$ feeds 42 Volts to PA1 thru PA8. Peak current through each Darlington is 20 Amperes: R1 thru R8 are meter shunts. The reference regulator (A15) provides the reference voltage for Q1 thru Q4a 
POWER SUPPLY - continued

Q6 is the +24 Volt regulator which is driven by a separate 28 Volt supply: 05 is a -24 volt regulator. It has its own AC power transformer T2. +28V current is 3 Amperes average.

\section{MAINTENANCE}

COOLING

Under no circumstances should the air flow be permitted obstruction for greater than ten minutes. The internal temperature of the heatsink and transistors may reach a critical stage, enabling the temperature protection circuit which will shut down operation. Reset is automatic after sufficient cooling has taken place.

\section{FUSES}

Fuses do not blow without cause: Before replacing the fuse, refer to schematics for proper power supply voltages. Also check the gating signal input to the amplifier for proper polarity and duty cycle.

To avoid damage to the unit; always replace with a fuse of equal value. 


\section{APPENDIX C}

\section{MACINTOSH SOFTWARE INSTRUCTIONS}




\section{Macintosh Software Instructions}

Before the Macintosh programs can be executed, the uscr must answer several requests such as filenames and the parameters associated with those files. This appendix lists the most common requests asked by the program plus some comments. In response to the requests, the uscr must type what follows in bold.

\section{readraw}

enter Input file name

coronal10

(user's choloe)

enter output file name

c.out

enter the number of blocks in the header

(user's choive)

28 for 3.2 and 54 for $3.2 \mathrm{~B}$

\section{8}

scan matrix $256 \quad 128$

no of echoes is 1

enter the spectral frequency in $\mathrm{MHz}$

20.5

enter the sweep width 2

10000

$1=1$

$1=2$

$j=3$

$j=4$

$J=5$

$1=6$

$1=7$

$1=8$

$1=9$

$J=10$

$j=11$ 


\section{$3 d|t c s|$}

enter output flle narine

4608.0ut

(user's choloe)

enter the matrlx dimenslons $(256 \times 16 \times 16)$

$256,16,16$

enter the sweep width

10000

enter the llne broadening

50

enter left shift number

0

enter flle name

4608.111

rinfo $=4.6007431 \mathrm{E}-40 \quad 10000.00 \quad 8.0447900 \mathrm{E}-38 \quad 8.0451476 \mathrm{E}-38$

$20.40000 \quad 8.1182584 E-38 \quad 8.1182590$ E-38 $\quad 8.1186155 E-38 \quad 256.0000$

256.0000

isize jsize $\quad 256 \quad 256$ 


\section{plotcsia}

enter Input flle name containing the $3 \mathrm{~d}$ ft csi output

\section{$2560.04 t$}

(user's choloe)

enter the spatlal row to start

1

enter the spatial column to start

1

enter the number of spectra to plot across

16

enter the rumber of spectra to plot down

16

enter the starting and ending chemical shift row to plot of each spectrum range 1.256

\section{1,256}

is this a raw data flle?

n

$\begin{array}{llll}\text { Ixsize } & 256 \quad 16 & 16\end{array}$

do you want to flip in the $x$ direction

n (y lf needed)

do you want to flip in the $y$ direction

n (y lf needed)

$\begin{array}{lc}\text { k in new coord } & 1 \\ \text { k in new coord } & 2 \\ \text { k in new coord } & 3 \\ \text { k in new coord } & 4 \\ \text { k in new coord } & 5 \\ \text { k in new coord } & 6 \\ \text { k in new coord } & ! \\ \text { k in new coord } & 8 \\ \text { k in new coord } & 9 \\ \text { k in new coord } & 10 \\ \text { k in new coord } & 11 \\ \text { k in new coord } & 12 \\ \text { k in new coord } & 13 \\ \text { k in new coord } & 14 \\ \text { k in new coord } & 15 \\ \text { k in new coord } & 16\end{array}$

PAUSE - Press RETURN to continue do you want to print this?

STOP 


\section{$2 d f t$}

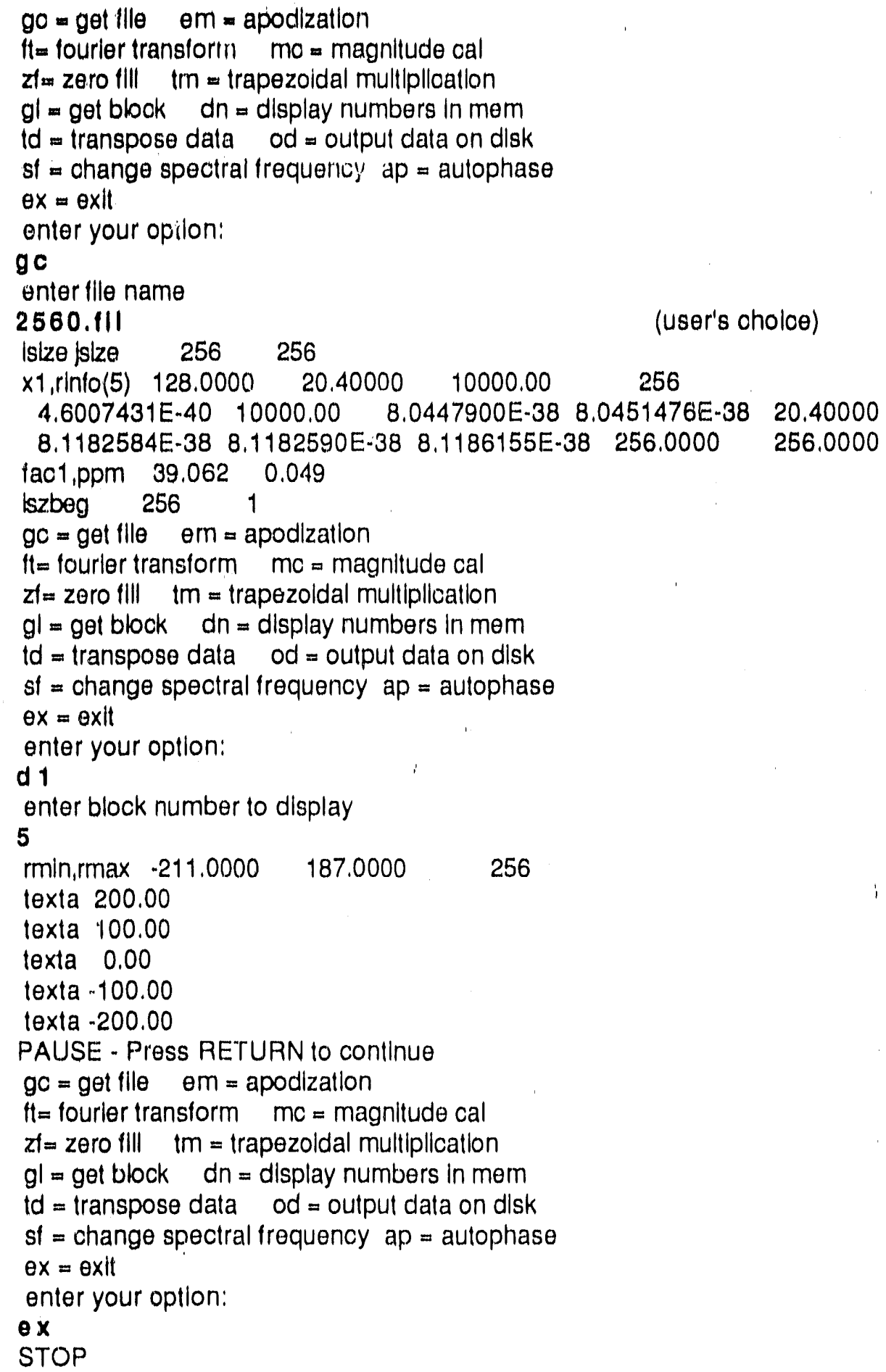


APPENDIX D

MR GLOSSARY

D. $1 / 2$ 


\section{MR Glossary}

Axial - A plane or slice in the X.Y plane, (Sce Magnot orientation.)

Chemical Shift - Minute, but measurable, changes in the Larmor frequency of specifie nuclel due to the effect of their chemical environment.

Chemical Shift Imaging - Information derived from nuclet when the data acquisition occurs without a readout gradient. Spectra for a single acquisition can be displayed with chomical shifts.

Coll - Single or multiple loops of wire designed either to produce a magnotic ficld from current flowing through the wire, or to detect a changing magnetic field by voltage induced in the wire.

Coronal - A plane or slice in the X. Z plane. (Sec Magnict orientation.)

Field of Vlew (FOV) - Acquisition ficld of view,

Fourier Transform - A mathomatical process for separating and analyzing individual frequencies from a multi-frequency signal. Mathematically this is defined as

$F(y)=\int_{-\infty}^{+\infty} f(x) e^{-2 x d x y} d x$

Free Induction Decay (FID) - Signal resulting after an RF pulse has excited the nuclear spin system to resonance. Called "free" since the nuclei precess frecly without applying RF.

Gauss - A measure of magnetic ficld strength (see Tesla).

Gradient Magnetic Field - A magnelic field which changes in strength in a certain given direction. Such fields are used in MR imaging to select a region for imaging and to encode the location of MR signals received from the region being imaged.

Gyromagnetic ratio $(\gamma)$ - Also reforred to as nuclear magnetogyric ratio. A constant for cach isotope. It is the ratio of angular frequency of precession to the magnetic field strength.

Homogeneity - Uniformity, In MR, the homogencity of the static magnetic ficld is an important critcrion of the quality of the magnet and the resulting images.

Larmor Frequency - The frequency at which nuclei begin to resonate when excited by a radiofrequency wave of the same energy, II is expressed in Herk, or cycles per second. $f=\gamma B_{0}$.

Longitudinal Relaxation - (also called "spin-lattice' relaxation) -- A mechanism through which some of the energy absorbed by the nuclei during RF excitation is dissipated to the chemical environment in which the nuclel are located. The time constant of this process is T1.

Magnetic Resonance (MR) - A physical phenomenon in which certain nuclei located in a strong, uniform magnetic field will absorb RF energy provided the frequency (f the exciting pulse matches the characieristic Larmor freguency of the nuclel present. 
Magnetic Vector $\mathbf{M}$ - The net bulk magnetization resulting from an entire population of nuclei.

Magnet Orientation - All spatial coordinates for imaging, etc., are referenced to the magnet providing the static magnetic $\mathrm{B}_{0}$ field. If the $\mathrm{B}_{0}$ field points out of the front of the magnet, the positive $\mathrm{Z}$ direction is in the same direction, positive $\mathrm{Y}$ is pointing up, and positive $"$ ' is pointing to the right.

Neutron - An uncharged subpotency particle found in all nuclei except in the most common hydrogen.

Nuclear Magnetic Resonance (NMR) - Same as MR. More commonly used in connection with spectroscopy.

Nucleus - The core of an atom, usually containing protons and neutrons.

Phantorn - An inert hody with a known structure and chemical composition which is used to calibrate the magnetic resonance imaging or spectroscopy process.

Precession - The gyration of axis of rotation.

Pulse Sequence - Sets of RF (and/or gradient) magnetic field pulses and time spacings thetween these pulses; used in conjunctior with gradient magnetic fields and MR signal reception to produce MR images.

Quadrature - A configuation in which two hodies have a separation of 90-degrees.

Relaxation - The physical means by which nuclei retum to equilibrium following excitation by RF energy, depending on either the interaction of the nuclei with their environment or with each ather.

Resonance - A physical phenomenon which permits the vansfer of energy from an object, particle or system, vibrating electrically or mechanically, to another similar object, particle or system, causing it to : wrate at the same frequency.

Sagittal - A plane or slice in the $Y-Z$ plane. (See Magnet orientation)

Spectroscopy - In MR, the ability to analyze chemical composition by detecting and quanufying changes in the Lamor frequencies of specific nuclei.

Spectrum - A graph showing the amplitudes of the components in an emission or absorption arranged in the ofder of some varying characteristic (such as frequency, mass, energy, etc.).

Surface Coil - A special RF receiver coil that can be placed over a region of interest for localization of sites during imaging or spectroscopy.

T1 - The relaxation time constant expressing the effect of the environment on nuclei.

T2 - The relaxation time constant expressing the effects of nuclei upon each other.

Tesla - A measurement of magnetic field strength ( 1 Tesla $=10,000$ Gauss). 
TR - Reposing time. Elapsed time between the beginning of one pulse sequence and the beginning of the succeeding (and usually identical) sequence.

Transverse Magnetic Field - A magnetic field created at right angles to the axis of a static magnetic field.

Transverse Relaxation - (also called 'spin-spin' relaxation) -- The process by which nuclei lose phase coherence following excitation by an RF signal in the presence of a static magnetic field. The time constant of this process is T2. 

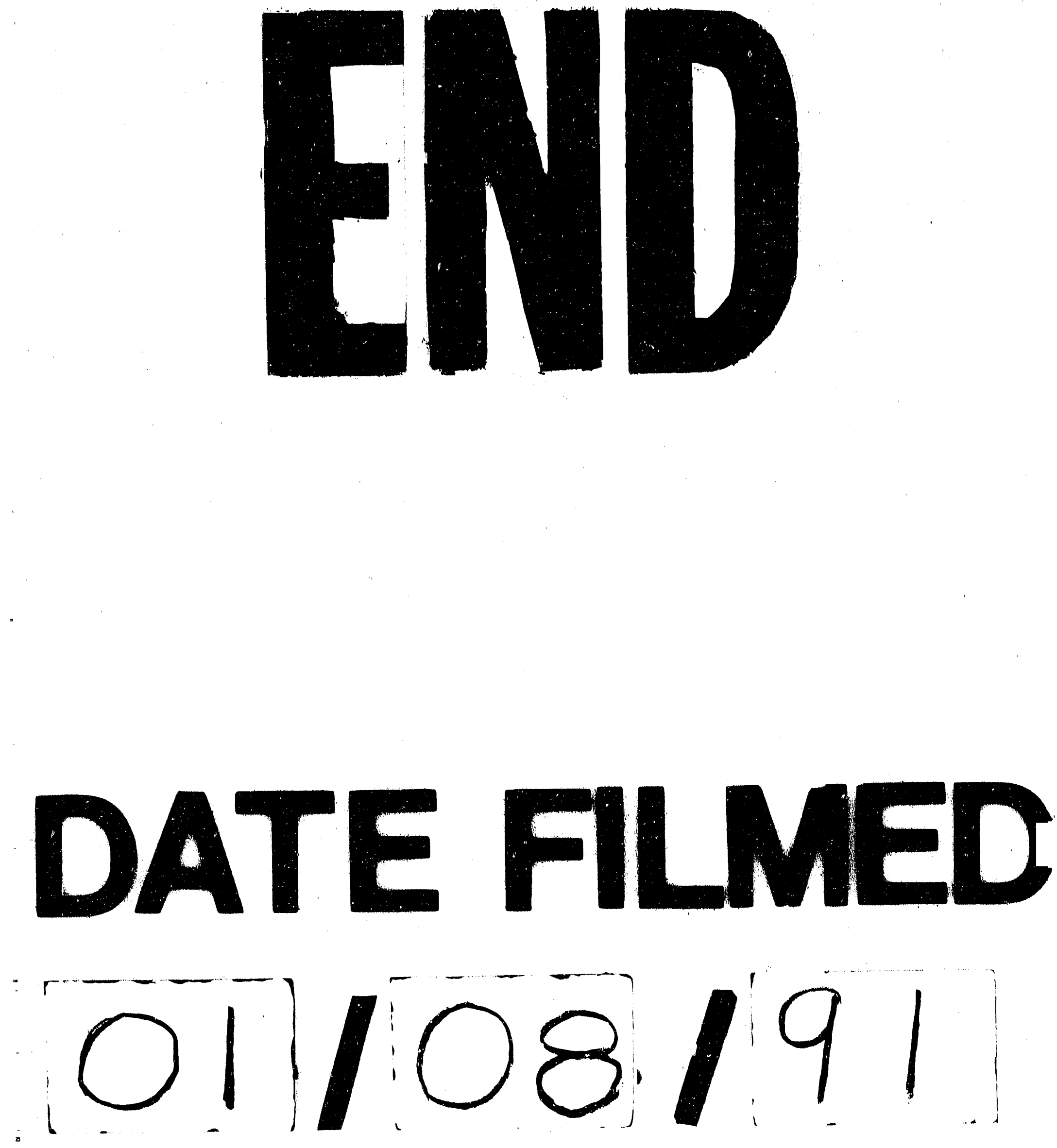
